\title{
An Empirical Examination of How Resilience Factors Mitigate the Effect of Childhood Adversity on Adolescent Mental Health
}

\author{
J. Fritz, ${ }^{1, *}$ J. Stochl, ${ }^{1,2}$ I.M. Goodyer, ${ }^{1}$ A.-L. van Harmelen, ${ }^{1, A}$ \& P.O. Wilkinson ${ }^{1, A}$ \\ * Correspondence: Jessica Fritz, if585@cam.ac.uk \\ ${ }^{1}$ University of Cambridge, Department of Psychiatry, United Kingdom \\ ${ }^{2}$ Charles University, Department of Kinanthropology, Czech Republic \\ A shared last author
}

\section{*Please note, this preprint is not yet peer-reviewed*}

Objective: More than one in three people worldwide are exposed to some form of childhood adversity (CA). CA is strongly associated with an increased risk for the development of mental health problems. Resilience factors (RFs), such as self-esteem, are known to reduce such vulnerability to mental health problems. Here we examine besides direct RF effects, whether RFs predominantly alter the relationship (i.e. moderation) or disrupt the developmental chain (i.e. mediation) between $\mathrm{CA}$ and mental health problems.

Methods: We studied 1130 adolescents. CA exposure and 10 RFs (3 inter-individual e.g. friendships; 7 intra-individual e.g. self-esteem) were assessed at age 14. Mental health problems were assessed at age 14 and 17, and were combined into a mental distress index. We estimated direct-effect, moderation and mediation models.

Results: When taking the effects of all $10 \mathrm{RFs}$ into account, five of the $10 \mathrm{RFs}$ had a significantly negative direct effect in the overall sample. However, of those five, only brooding revealed a significant effect in both the group of adolescents with and without CA. Of the 10 RFs, friendship support, family cohesion and distress tolerance had a significantly negative relationship with age-17 distress in the group of adolescents with CA, and no effect in the group of adolescents without $\mathrm{CA}$. Yet, none of the three RFs revealed a significant moderation effect $(p=0.07-0.09)$. Positive self-esteem, negative self-esteem and aggression functioned as significant mediators for the relationship between CA and mental distress.

Conclusions: In situations in which CA is not known, brooding seems to be the most beneficial $\mathrm{RF}$, as it reduced subsequent mental distress in both adolescents with and without CA. Regarding RFs in the aftermath of CA, our findings provide predominant support for mediation rather than for moderation effects. More specifically, targeting negative self-esteem, positive self-esteem, and aggression seems most fruitful, when the aim is to disrupt the chain between $\mathrm{CA}$ and adolescent mental health problems.

Keywords: childhood adversity, resilience factors, protective factors, resource factors, psychopathology, distress, moderation, mediation 


\section{An Empirical Examination of How Resilience Factors Mitigate the Effect of Childhood Adversity on Adolescent Mental Health}

Childhood adversity (CA) is often assumed to be a rare incidence. ${ }^{1}$ However, more than one in three people worldwide are exposed to some form of adversity prior to the age of $18 .^{2}$ Several large-scale epidemiological studies even reported ratios as high as one in two people., Generally, CA refers to a single traumatic event (e.g. death of a significant other), or to several severely stressful events (e.g. parental mental illness with significant impact on the family life). According to the CA definition put forward by Katie McLaughlin (2016), ${ }^{5}$ CA either comprises a notable divergence of a reasonable living situation, or a notable alteration of the anticipated living situation, and thus demands active adjustment by the child or adolescent. ${ }^{5}$ Therefore, CA is likely to impact social, emotional, behavioural and cognitive functioning and development. ${ }^{5}$ The justification of treating $\mathrm{CA}$ as an aggregated index, rather than as separate forms of traumatic events or severe stress, stems from a solid body of research showing that different forms of CA often co-occur and that most forms are strongly associated with subsequent mental health problems. ${ }^{2-5} \mathrm{CA}$ has been found to increase the risk of subsequent mental health problems particularly proximally after the exposure, but it is also found to have a lasting impact across the life span. ${ }^{2,3}$ Therefore, it is crucial that we better understand those factors that alleviate the risk and development of mental health problems following CA, so called resilience factors (RFs). ${ }^{6}$

Simply put, RFs are factors that improve or stabilize mental health in the aftermath of adverse experiences. In our preregistered systematic review ${ }^{6}$ we therefore defined RFs as those factors that moderate and/or mediate the relationship between CA and subsequent mental health problems. Importantly, we only included those RFs that function on a social, emotional, cognitive or behavioural level, to ensure that RFs are theoretically amenable to intervention. ${ }^{6}$ Here, we investigate three inter-individual (friendship support, family support, family cohesion) and seven intra-individual RFs (positive self-esteem, negative self-esteem, brooding, reflection, distress tolerance, aggression potential, and expressive suppression) that were empirically supported in our systematic review ${ }^{6}$ and assessed in our adolescent cohort. ${ }^{7}$ Previous research on those 10 RFs has shown that the RFs function as a protective system in which RFs enhance each other less in adolescents with CA than in adolescents without CA. ${ }^{8}$ Moreover, we showed that most of the 10 RFs were not only concurrently associated with less mental health problems, but also three years later. ${ }^{89}$ More specifically, the RFs predicted mental distress three years later similarly well as mental distress could predict itself. ${ }^{9}$ Hence, there is good support showing that most of the 10 RFs do indeed reduce distress; or in other words, that RFs have a direct, negative effect on mental health problems. However, as of yet, it is unclear how RFs decrease the detrimental consequences CA poses on mental health. Therefore, we here aim to investigate whether RFs function predominantly as moderators or as mediators for the positive relationship between CA and subsequent mental health problems. This is important, as moderating and mediating RFs differ theoretically, statistically, and with regard to the inferences that can be drawn..$^{10,11}$

Theoretically, moderating RFs are RFs that modify the way CA is associated with subsequent mental health problems. That is, the RF operates on the relationship between CA and mental health as it reduces mental health problems more in adolescents with (CA+) than in adolescents without CA (CA-). Taking self-esteem as example, self-esteem would need to reduce mental health problems more in $\mathrm{CA}+$ than in CA- adolescents. Or to put it differently, the negative effect of CA on mental health problems would need to be stronger for adolescents with a low level of self-esteem than for adolescents with a high level of self-esteem. This is depicted in Figure 1a1. For such an effect, CA and the RF should be assessed before the mental 
health outcome; CA, however, does not necessarily need to have happened prior in time to the $\mathrm{RF}$ assessment. Rose and colleagues ${ }^{12}$ refer to such RFs as "protective factors". For mediation effects, a timeline is crucial. Here the RF is not meant to directly modify the relationship between CA and mental health problems, but is supposed to be the intermediate factor explaining this relationship (at least in part). That is, CA is expected to lead to a lower level of the RF, while a higher level of the RF is expected to lead to less subsequent mental health problems. As mediating RFs are expected to function as intermediate factors, the RF should timewise be assessed in the aftermath of CA, but before the mental health problems. Taking again self-esteem as example, CA is expected to lead to lower self-esteem, while higher selfesteem is expected to lead to less subsequent mental health problems. This is depicted in Figure 1b1. Rose and colleagues ${ }^{12}$ refer to such RFs as "resource factors".

\section{Theoretical model}

(a1)

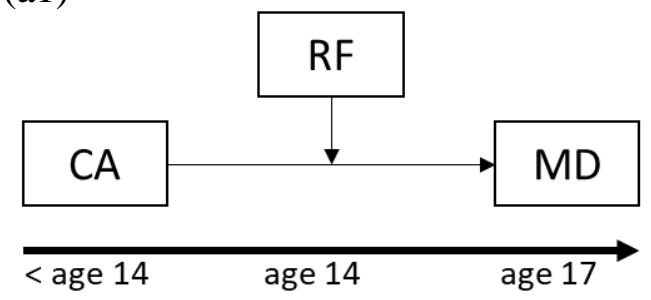

(b1)

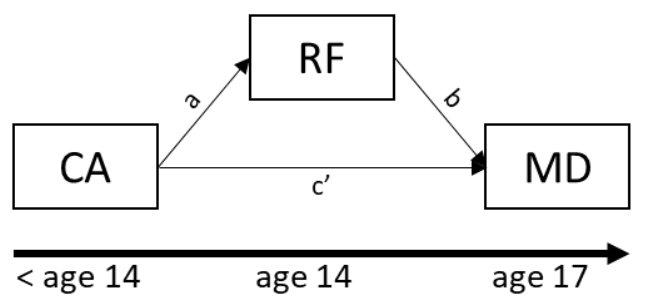

\section{Statistical model}

(a2)

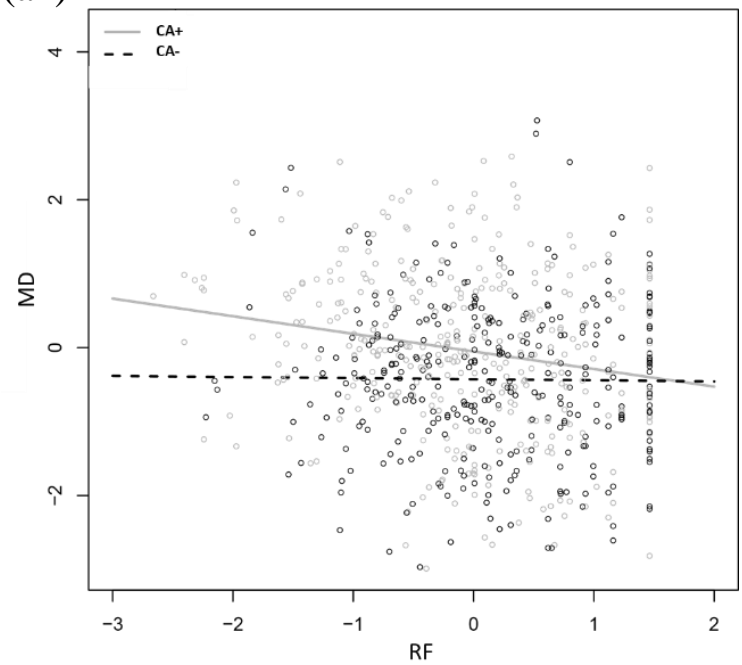

(b2)

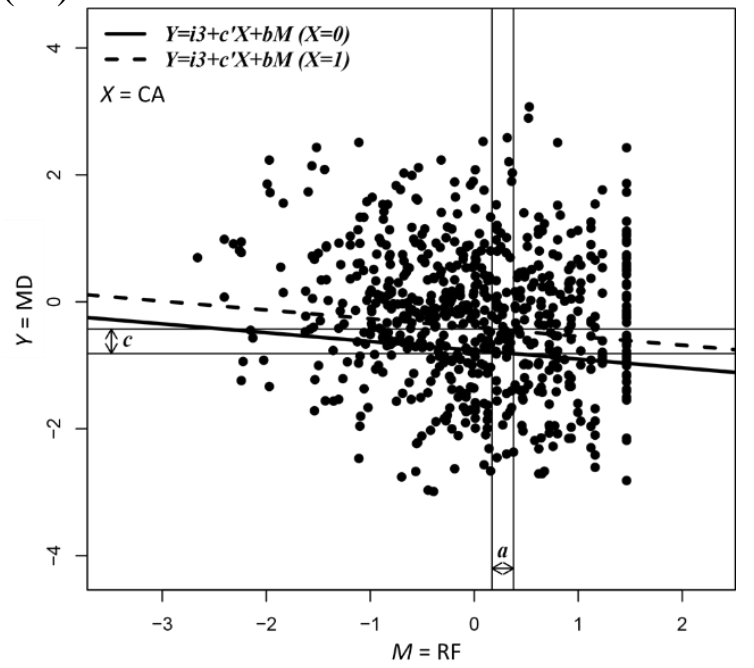

Figure 1. $\mathrm{CA}=$ childhood adversity, $\mathrm{RF}=$ resilience factors, $\mathrm{MD}=$ mental distress $/$ mental health problems. (a1) $=$ theoretical model for a single moderation effect, $(\mathrm{b} 1)=$ theoretical model for a single mediation effect, $(\mathrm{a} 2)=$ statistical model for a single moderation effect, (b2) = statistical model for a single mediation effect. Model (b1): the a-path represents the effect of CA on the RF; the b-path represents the effect of RFs on MD, while taking the effect of CA on MD into account; the c'-path represents the effect of CA on MD, while taking the effect of RFs on MD into account; the c-path, which is not directly depicted, would represent the total effect of CA on MD, without taking the effect of RFs into account; the a*b effect, which is not directly depicted, would represent the indirect effect of CA on MD, via the RF. Model (b2): the letters represent the same paths/effects as in the theoretical model in (b1); the dashed regression line depicts the line for adolescents with $\mathrm{CA}(\mathrm{CA}+)$ and the solid regression line the line for adolescents without $\mathrm{CA}(\mathrm{CA}-)$; c denotes the total effect $\mathrm{CA}$ poses on $\mathrm{MD}$, with the difference representing the mean level difference in MD for CA+ (upper line) and CA- (lower line) adolescents (taking into account the stable intercept); a denotes the total effect CA poses on RFs, with the difference representing the mean level difference in RFs for $\mathrm{CA}+$ (left line) and CA- (right line) adolescents (taking into account the stable intercept); $\mathrm{b}$ is represented by the slope of the CA+ as well as the CA- regression line; c' is represented by the distance between the solid CA- regression line and the horizontal line for CA+ adolescents on MD (upper line) located on the vertical CA+ line for RFs (left vertical line); $a^{*} b$ is represented by the distance between the horizontal line for CA- adolescents on MD (lower line) and the solid CA- regression line, located on the vertical CA+ line for RFs (left vertical line). Information regarding the code with which the figure in panel (b2) was create can be found in Supplement II. 
Statistically, a moderating RF is supposed to reduce subsequent mental health problems more in $\mathrm{CA}+$ than in CA- adolescents. This is reflected in the regression slope of the RF, which should be significantly steeper in the CA+ than the CA- group. Such an effect is also called an interaction effect and is visualized in Figure 1a2. In contrast, a mediating RF can reduce distress equally in the CA+ and CA- adolescents, as long as the CA exposure results in a lower level of the RF, and a higher level of the RF results in a lower level of mental health problems. Therefore, the regression lines of the RF do not need to differ in steepness between the two groups; but, the regression slope in the $\mathrm{CA}+$ group should reflect a higher level of mental health problems than in the CA-group, at the same level of the RF. This is also called an indirect effect and is visualized in Figure 1b2. Importantly, an RF which reduces mental health problems more in the $\mathrm{CA}+$ than in the $\mathrm{CA}$ - group can at the same time be a moderator and a mediator (provided that $\mathrm{CA}$ increases subsequent mental health problems, $\mathrm{CA}$ is negatively associated with the succeeding RF, and the RF reduces subsequent mental health problems when the effect of CA is taken into account).

Regarding inferences, moderating RFs can be considered as mainly advantageous for $\mathrm{CA}+$ adolescents as they reduce distress more in CA+ than CA- group. Mediating RFs can be considered as intermediate, alleviating effect on the pathway from CA to subsequent mental health problems. In this sense, increasing the level of the RF can lead to improved mental health particularly in adolescents with CA (moderator) and/or break or disrupt the chain between CA and mental health problems (mediator). Therefore, both moderating and mediating RFs can serve as prevention and treatment targets for people who have been exposed to CA. Moreover, some RFs reduce subsequent distress significantly for both $\mathrm{CA}+$ and $\mathrm{CA}$ - adolescents. A notable advantage of those RFs is that they can serve as prevention or treatment targets for the entire population; for instance, when it is inappropriate or impossible to assess an adversity history or when adolescents with and without CA need to be treated equally. Overall, we believe that shedding light on effects that describe whether and how RFs reduce mental health problems after CA not only advances empirical knowledge for mental health promotion ${ }^{10,12}$ and theoretical understanding of protective effects in the face of adversity, ${ }^{11,12}$ but could also directly inform translational efforts aimed at reducing mental health problems..$^{10,12}$

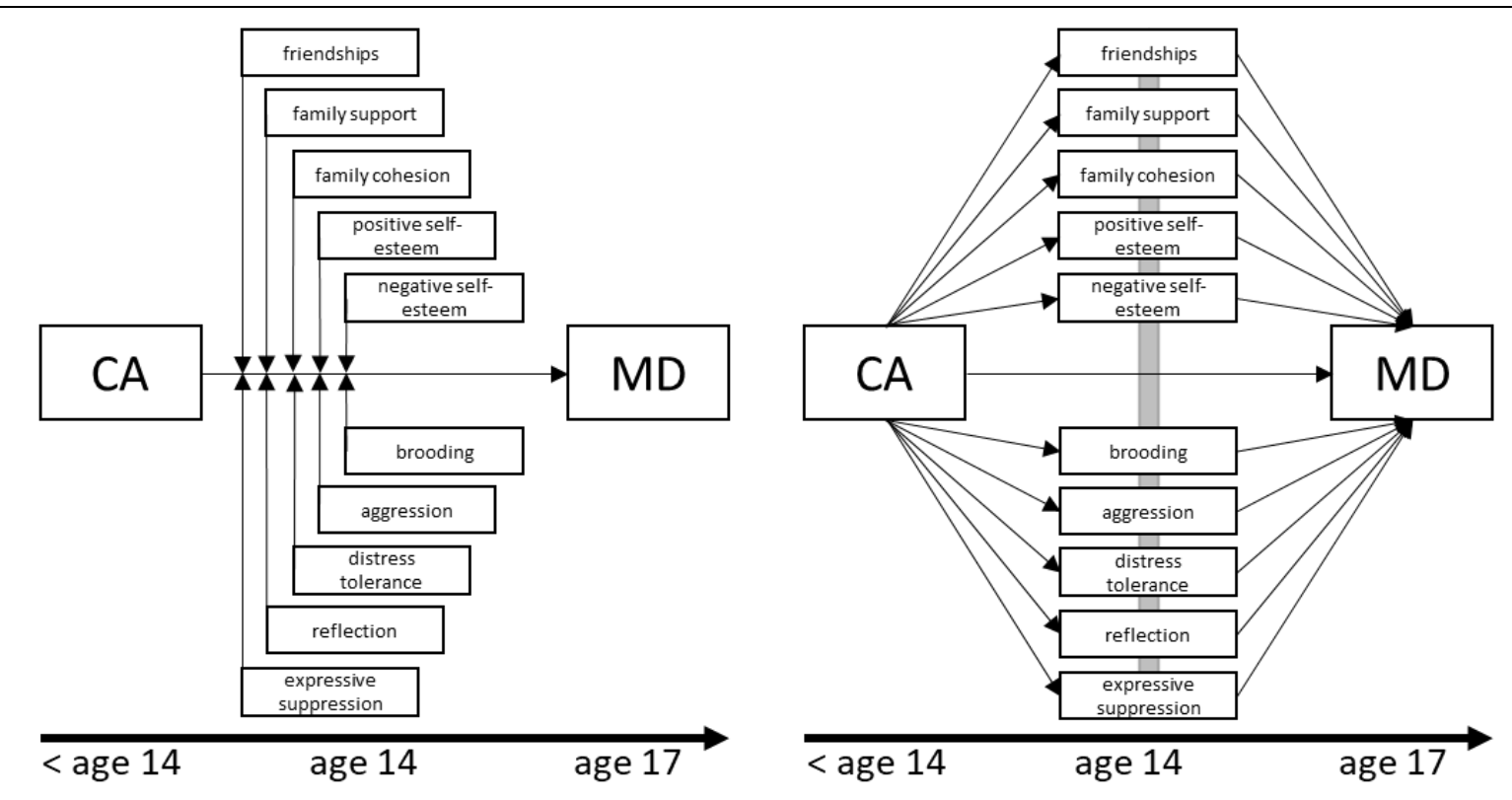

Figure 2. CA+ childhood adversity, MD = mental distress/ mental health problems. The left panel depicts a multiple moderator model and the right panel the multiple mediator model, both including all RFs. The grey bar in the mediation model indicates that the RFs are allowed to correlate with each other. 
In sum, besides exploring direct effects of RFs on subsequent mental health problems, we will shed light on the question which RFs function as moderator and/or as mediator for the relationship between $\mathrm{CA}$ and subsequent mental health problems. We investigate this in two ways, firstly by examining single RFs, i.e. "single RF models" (see Figure 1), and secondly by examining the RFs while taking the effects of the remaining RFs into account, i.e. "multiple RF models" (see Figure 2). Investigating multiple RF models is important, to safeguard overestimating single RF effects and to enhance ecological validity.

\section{METHODS}

\section{Design}

In 2005 and 2006, 1238 adolescents were recruited for the ROOTS study, in schools in and around Cambridgeshire (UK). ${ }^{7}$ For inclusion the adolescents had to be 14 years old. The adolescents and their main caregiver provided written informed consent. The adolescents were followed up at age $17 .{ }^{7}$ Here we include 1130 adolescents for whom we have complete data for CA before age 14, and could impute data for age-14 RFs, and distress at both age 14 and 17. ROOTS was conducted along the lines of the Declaration of Helsinki, in accordance with Good Clinical Practice guidelines and was approved by the Cambridgeshire Research Ethics Committee (03/302). ${ }^{13}$

\section{Childhood Adversity (CA)}

Adversity before the age of 14 was assessed with the semi-structured Cambridge Early Experiences Interview (CAMEEI), and was performed with the primary caregiver. ${ }^{713}$ The CAMEEI assessed several different types of CA: family discord, separation for more than six months, living in foster care, lack of maternal affection, lack of maternal engagement, inappropriate parenting, death of a significant other, parental criminality, violence within the family, significant social difficulties within the family, significant and/or chronic mental or physical illness of a parent or sibling, significant external disturbances (e.g. effects of war), significant environmental disturbances (e.g. fire), financial difficulties (with and without parental unemployment), and emotional, physical and/or sexual abuse. Dunn and colleagues ${ }^{13}$ conducted a latent class analysis and clustered the adolescents into no CA, aberrant parenting $\mathrm{CA}$, moderate $\mathrm{CA}$ and severe $\mathrm{CA}$. The clustering was performed for the time-windows from zero to five, five to eleven, and eleven to fourteen years, which were used during the assessment of the CAMEEI to enhance recall. Consistent with previous reports we here clustered adolescents into $\mathrm{CA}$-, which is no $\mathrm{CA}$ for all three time-windows, and $\mathrm{CA}+$, which is either of the three types of CA for at least one of the three time-windows. ${ }^{14}$ A detailed exploration of the CA subtypes can be found in Dunn and colleagues (2011) report. ${ }^{13}$

\section{RFs}

Ten RFs were assessed with questionnaires at age 14. All those RFs were identified by our preregistered systematic review ${ }^{6}$ and are amenable, so that the focus lies exclusively on those RFs that are empirically supported and can theoretically, directly be targeted in psychological interventions. The first eight RFs are assessed via adolescent report, the last two via caregiver report:

1. High levels of high friendship support are considered protective and were assessed with five items of the Cambridge Friendships Questionnaire. ${ }^{15}$

2. High levels of high family support are considered protective and were assessed with five items of the McMaster Family Assessment Device. ${ }^{16}$ 
3. High levels of high family cohesion are considered protective and were assessed with the remaining seven items of the McMaster Family Assessment Device. ${ }^{16}$

4. High levels of low negative self-esteem are considered protective and were assessed with five items of the Rosenberg self-esteem scale (required reversion of items). ${ }^{17}$

5. High levels of high positive self-esteem are considered protective and were assessed with the remaining five items of the Rosenberg self-esteem scale. ${ }^{17}$

6. High levels of low ruminative brooding are considered protective and were assessed with five items of the Ruminative Response Scale (RRS; required reversion of items). ${ }^{18,19}$

7. High levels of low reflective rumination are considered protective and were assessed with five other items of the RRS (required reversion of items). ${ }^{18,19}$

8. High levels of high distress tolerance are considered protective and were assessed with five items of the Emotionality Activity Sociability Temperament Survey. ${ }^{20}$

9. High levels of low aggression are considered protective and were assessed with four items of the Behaviour Checklist (11 questions based on the DSM-IV criteria for conduct problems; required reversion of items). ${ }^{21,22}$

10. High levels of low expressive suppression are considered protective and were assessed with one item of the Antisocial Process Screening Device (required item reversion). ${ }^{23}$

Psychometric information for the RF measures can be found in Supplement XIII in Fritz, Fried et al. ${ }^{14}$

\section{Mental distress}

A latent distress factor was estimated based on 13 depression related items, measured with the Short Mood and Feelings Questionnaire, ${ }^{24}$ and 28 anxiety related items, measured with the Revised Children's Manifest Anxiety Scale. ${ }^{25}$ Higher scores on the latent distress factor indicate higher levels of internalizing-related mental health problems. ${ }^{14}$ In adolescent cohorts, latent distress factors have been found to replicate soundly. ${ }^{26-28}$

\section{Analysis}

Missing data patterns on the RFs and the distress items could be explained by several demographic and life-history variables, such as gender, a CA history, and a prior psychiatric history (for details see Fritz, Stochl, et al., 2019a $a^{8}$ : Additional file IV Table 3). Therefore, we decided to impute missing data for all RF (103 items) and distress items (122 in total; 41 items +20 additional items of the full scale for depression related symptoms, for both ages, to include as much information as possible). We used the aforementioned demographic and life-history variables, as well as age and socio-economic status (for details see Table 1), as additional auxiliary variables ( 7 items). We decided not to impute data for the CA variable, as some types of CA did not seem sufficiently predictable (e.g. a fire in the home or a traumatic car crash). Ordinal items were imputed with predictive mean matching and binary items via logistic regression, using multivariate multiple imputation algorithms with chained equations (using 100 iterations). ${ }^{29}$ We excluded participants who had more than $85 \%$ missing items. Eventually, we could compute 10 complete data sets for 1188 participants. Based on this data we computed the RFs using categorical, confirmatory factor analyses, with a weighted least squares mean and variance corrected (WLSMV) estimator. Similarly, we conducted a strongly invariant categorical confirmatory factor analysis, using the WLSMV estimator and a logit link, for the distress variable. The invariant model was identified in line with $\mathrm{Wu}$ and Estabrook $^{30}$ (see Supplement I in Fritz Stochl et al., 2019b ${ }^{9}$ ). We decided to use factor scores rather than sum scores to avoid tau-equivalence and to reduce measurement error (for a rationale see Additional file V Part A in Fritz, Stochl et al., 2019 $\mathrm{a}^{8}$ ). We pooled over the resulting factor scores and fit 
indices and extracted the resulting pooled factor scores for further analyses (see Supplement I in Fritz Stochl et al. $2019 b^{9}$ for details). In sum, we could conduct the main analyses on 1130 participants, as those could be included in the imputation analyses $(n=1188)$ and had data for CA $(n=1139)$. For completeness, we provide results based on non-imputed data in the Additional Supplement File. Next, we set out to conduct two batches of analyses.

Firstly, we aimed to shed light on the question of which RFs function (on their own) as direct effect, moderator and/or mediator for the relationship between CA and subsequent distress. To this end we started examining whether all age-14 RFs were significantly negatively associated with age-17 distress in (a) the whole sample, (b) the CA+ group, as well as (c) the CA-group. A negative association at least in the $\mathrm{CA}+$ group should be a prerequisite regardless of the effect being a moderation or mediation effect. We then conducted "single" mediator and moderator models, each with one RF. For the moderation models we computed linear regression analyses with the respective age-14 RF, CA and the RF-CA interaction term (i.e. the RF multiplied with CA) as independent variables, and age-17 distress as dependent variable, to assess whether the RF moderates the relationship between CA and age-17 distress. ${ }^{11}$ As most RFs and distress were derived from factor analyses the variables were centered at zero. To facilitate the interpretation of the binary aggression and expressive suppression RFs, we also centered those variables at zero. For the mediation models we first confirmed that the direct effect from CA on age-17 distress was significant. We then estimated a path model with CA predicting the age-14 RF (a-path) as well as age-17 distress (c'-path), and with the age-14 RF predicting age-17 distress (b-path; hence the c'-path is corrected for the effect of the RF and the b-path is corrected for the effect of CA). We additionally tested the significance of the indirect effect, which was based on the multiplication of the a-path and the

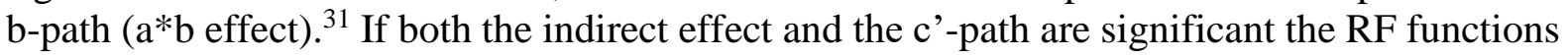
as partial mediator. If the indirect effect is significant, but the c'-path is not, then the RF is a full mediator (provided that the single direct effect had been significant). The mediation models were conducted in three ways, to evaluate and ensure robustness: (1) with a maximum likelihood (ML) estimator and 1000 bootstraps for the standard errors, ${ }^{32}$ (2) with a robust maximum likelihood estimator (MLR; to compute robust standard errors as described by Huber-White and scaled test statistics along the lines of Yuan-Bentler), ${ }^{33}$ and (3) with an ML estimator and simulated Monte Carlo confidence intervals. ${ }^{32}$ Moreover, we applied the false discovery rate for the single RF models, to correct for multiple testing effects.

Secondly, we again aimed to investigate the question which RFs function as direct effect, moderator and/or mediator for the relationship between CA and subsequent distress, while this time taking the effects of the remaining RFs into account (i.e. "multiple" regression, mediator and moderator models). To this end we started examining whether all age-14 RFs were significantly negatively associated with age-17 distress in (a) the whole sample, (b) the $\mathrm{CA}+$ group, as well as (c) the CA- group, while correcting for all other RFs. For the moderation analyses we entered all age-14 RFs, CA and all RF-CA interaction terms (i.e. 10 interactions in total) as independent variables, and age-17 distress as dependent variable to the regression model, to assess which RFs moderate the relationship between CA and age-17 distress. For the multiple mediation analysis we estimated a path model in which CA predicted the age-14 RFs (a-paths) as well as age-17 distress (c'-path), and in which all age-14 RFs predicted age-17 distress (b-paths; hence the c'-path is corrected for the effects of the RFs and the b-paths are corrected for the effect of CA). The RFs were allowed to correlate with each other. We tested the significance of each indirect RF effect based on the multiplication of the respective a- and b-path ( $a^{*} \mathrm{~b}$ effect). Moreover, we calculated a cumulative indirect effect adding up single indirect RF effects. Importantly, for the cumulative indirect RF effect we only included RFs that had a negative relationship with both CA and age-17 distress, when taking all other RFs into account, as only those can function as significant RF mediators. ${ }^{6}$ The mediation models 
were again conducted (1) with an ML estimator and 1000 bootstraps for the standard errors, ${ }^{32}$ (2) with an MLR estimator, ${ }^{33}$ and (3) with an ML estimator and simulated Monte Carlo confidence intervals. ${ }^{32}$

Importantly, all analyses were performed and will be reported with correction for gender, as gender has been shown to have a critical effect on distress. ${ }^{26}$ The results without correction for gender are reported in Supplement II. Most analyses were performed in R 3.5.1 ${ }^{34}$ and all used packages and version numbers can be found in Supplement III. The invariant categorical factor analysis for distress was performed in Mplus 8.2. ${ }^{35}$ Details regarding power considerations can be found in Supplement IV.

\section{Data availability}

Data for this specific paper has been uploaded to the Cambridge Data Repository https://doi.org/10.17863/CAM.46642 and is password protected. Our participants did not give informed consent for their measures to be made publicly available, and it is possible that they could be identified from this data set. Access to the data supporting the analyses presented in this paper will be made available to researchers with a reasonable request to openNSPN@medschl.cam.ac.uk.

\section{Code availability}

Analysis code is available on www.jessica-fritz.com.

\section{RESULTS}

\section{Sample}

Of the 1238 ROOTS participants 1139 had complete data for CA prior to age 14, and 1188 could be included in the imputation analyses for the age-14 RFs and general distress at age 14 and 17 (as they had less than $85 \%$ missing data), resulting in total in 1130 adolescents for whom we could compute the analyses $(C A+n=631, C A-n=499$; female $=620$, male $=510)$. Both gender and age did not differ between the $\mathrm{CA}+$ and the CA- group; but $\mathrm{CA}+$ adolescents had more often a prior psychiatric history and a lower socio-economic status (SES; see Table 1). Age and SES did not differ between males and females, but male adolescents less often had a prior psychiatric history.

Table 1

Sample description, split for CA and gender

\begin{tabular}{|c|c|c|c|c|}
\hline & $\mathrm{CA}+(\mathrm{n}=631)$ & $\mathrm{CA}-(n=499)$ & $\chi^{2} / z / t(d f)$ & p-value \\
\hline \multirow[t]{2}{*}{ gender } & Female $=358$ & Female $=262$ & $1.85(1)$ & .17 \\
\hline & Male $=273$ & Male $=237$ & & \\
\hline age $14^{*}$ & $14.49(0.28)$ & $14.48(0.28)$ & $-0.43(1049.3)$ & .67 \\
\hline age $17 *$ & $17.49(0.34)$ & $17.48(0.32)$ & $-0.48(1015.8)$ & .63 \\
\hline \multirow[t]{5}{*}{ SES } & Hard pressed $=73$ & Hard pressed $=30$ & 5.24 & $<.001 *$ \\
\hline & Moderate means $=36$ & Moderate means $=11$ & & \\
\hline & Comfortably off $=168$ & Comfortably off $=104$ & & \\
\hline & Urban prosperity $=37$ & Urban prosperity $=41$ & & \\
\hline & Wealthy achievers $=317$ & Wealthy achievers $=313$ & & \\
\hline prior psychiatric & Yes $=199$ & Yes $=74$ & $41.54(1)$ & $<.001 *$ \\
\hline history at age 14 & No $=432$ & $\mathrm{No}=425$ & & \\
\hline prior psychiatric & Yes $=267$ & Yes $=122$ & $48.05(1)$ & $<.001 *$ \\
\hline \multirow[t]{2}{*}{ history at age $17^{*}$} & No $=297$ & $\mathrm{No}=345$ & & \\
\hline & Female $(n=620)$ & Male $(n=510)$ & $\chi^{2} / z / t(d f)$ & p-value \\
\hline age $14 *$ & $14.49(0.27)$ & $14.48(0.29)$ & $0.61(1027)$ & .54 \\
\hline age $17 *$ & $17.50(0.32)$ & $17.47(0.34)$ & $1.38(954.71)$ & .17 \\
\hline
\end{tabular}




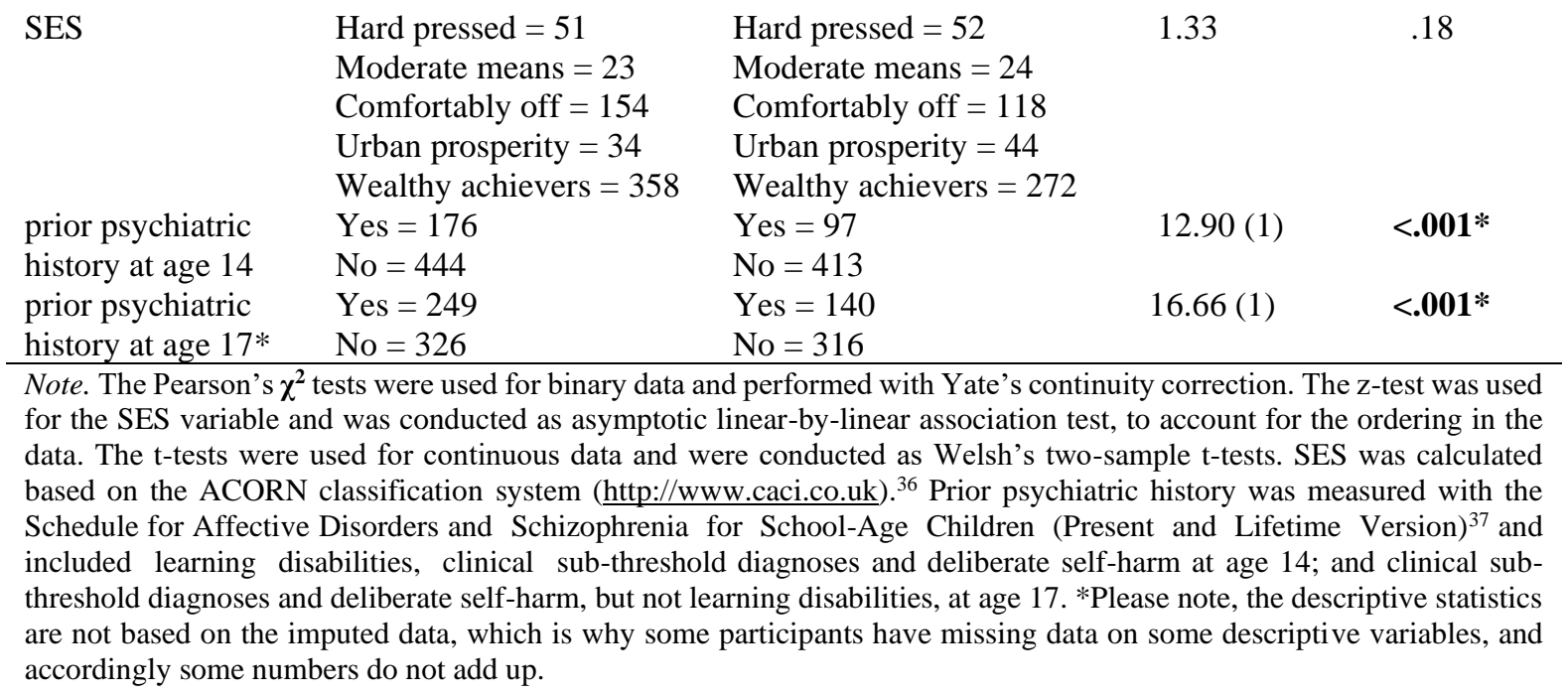

\section{Direct, moderation and mediation effects for single RF models}

Direct effects. Eight of the 10 RFs were significantly negatively associated with age17 distress, in the CA+ group (Table 2 middle panel), the CA- group (Table 2 right panel), as well as in the whole sample (Table 2 left panel). Distress tolerance revealed a significantly negative effect in the CA+ group and the whole sample, but not in the CA-group. Expressive suppression was not associated with age-17 distress in any of three (sub)groups.

Table 2

Single main effects of the RFs with correction for gender, for the whole sample, the CA+ and the CAgroup; corrected for multiple testing

\begin{tabular}{|c|c|c|c|c|c|c|c|c|c|c|c|c|c|}
\hline & \multicolumn{5}{|c|}{ Whole sample } & \multicolumn{4}{|c|}{$C A+$} & \multicolumn{4}{|c|}{ CA- } \\
\hline & $\mathrm{b}$ & $\mathrm{b}^{*}$ & SE & $\mathbf{p}$ & $\mathrm{R}^{2}$ & $\mathrm{~b}$ & SE & $\mathbf{p}$ & $\mathrm{R}^{2}$ & $\mathrm{~b}$ & SE & $\mathbf{p}$ & $\mathrm{R}^{2}$ \\
\hline $\mathrm{CA}$ & 0.43 & - & .08 & $<0.001 *$ & 04 & - & - & - & - & - & - & - & - \\
\hline Frn & -0.35 & -0.33 & .05 & $<0.001 *$ & 06 & -0.35 & .06 & $<0.001 *$ & 06 & -0.29 & .07 & $<0.001 *$ & 06 \\
\hline Fms & -0.31 & -0.29 & .04 & $<0.001 *$ & 06 & -0.28 & .06 & $<0.001 *$ & 04 & -0.31 & .06 & $<0.001 *$ & 07 \\
\hline Fmc & -0.38 & -0.34 & .04 & $<0.001 *$ & 08 & -0.37 & .06 & $<0.001 *$ & 07 & -0.30 & .07 & $<0.001 *$ & 06 \\
\hline Pst & -0.45 & -0.43 & .04 & $<0.001 *$ & 12 & -0.42 & .05 & $<0.001 *$ & 09 & -0.45 & .06 & $<0.001 *$ & 12 \\
\hline $\mathrm{Ngt}$ & -0.58 & -0.56 & .05 & $<0.001 *$ & 14 & -0.49 & .06 & $<0.001 *$ & 10 & -0.67 & .07 & $<0.001 *$ & 18 \\
\hline Brd & -0.48 & -0.47 & .04 & $<0.001 *$ & 11 & -0.44 & .06 & $<0.001 *$ & 09 & -0.50 & .06 & $<0.001 *$ & 13 \\
\hline Rfl & -0.29 & -0.28 & .05 & $<0.001 *$ & 05 & -0.25 & .07 & $<0.001 *$ & 03 & -0.31 & .07 & $<0.001 *$ & 06 \\
\hline Dst & -0.24 & -0.21 & .04 & $<0.001 *$ & 04 & -0.29 & .06 & $<0.001 *$ & 05 & -0.09 & .07 & 0.18 & 03 \\
\hline Agg & -0.28 & -0.26 & .04 & $<0.001 *$ & 06 & -0.24 & .05 & $<0.001 *$ & 05 & -0.28 & .06 & $<0.001 *$ & 06 \\
\hline Exp & 0.00 & 0.02 & .04 & 0.98 & 02 & 0.04 & .05 & 0.42 & 01 & -0.01 & .06 & 0.81 & 02 \\
\hline D14 & 0.65 & 0.63 & .04 & $<0.001 *$ & 22 & 0.61 & .05 & $<0.001 *$ & 19 & 0.66 & .06 & $<0.001 *$ & 25 \\
\hline
\end{tabular}

Note. $\mathrm{b}=$ unstandardized regression (slope) coefficient, $\mathrm{b}^{*}=$ unstandardized regression (slope) coefficient corrected for CA; $\mathrm{SE}=$ standard error, $\mathrm{p}=\mathrm{p}$-value, $\mathrm{R}^{2}=$ variance explained, represented here as percentage. Of note, $\mathrm{SE}, \mathrm{p}$ and $\mathrm{R}^{2}$ belong to the effects that are not corrected for $\mathrm{CA} . \mathrm{CA}=$ childhood adversity, $\mathrm{CA}+=$ adolescents with $\mathrm{CA}, \mathrm{CA}-=$ adolescents without $\mathrm{CA}$; Frn = friendship support Fms = family support Fmc = family cohesion; $P$ st $=$ positive self-esteem; Ngt = negative self-esteem; Brd = brooding; Rfl = reflection; Dst $=$ distress tolerance; Agg = aggression; Exp = expressive suppression; D14 = age-14 distress. The belonging results that are not corrected for gender can be found in Supplement II.

Moderation. None of the 10 RFs functioned as a moderator for the relationship between $\mathrm{CA}$ and age-17 distress (see Table 3).

Mediation. Eight of the 10 RFs functioned as significant RF mediators for the relationship between CA and age-17 distress (see Table 3), as for all those RFs CA was associated with a lower level of the RF and the RF in turn was associated with a lower level of age-17 distress. Reflection did not function as a mediator, as CA was not associated with a 
lower level of reflection. Expressive suppression did not function as a mediator as it was not associated with age-17 distress. Importantly, all mediating RFs functioned as partial mediators.

Table 3

Single moderation and mediation effects of the RFs with correction for gender; corrected for multiple testing

\begin{tabular}{|c|c|c|c|c|c|c|c|c|}
\hline & \multicolumn{4}{|c|}{ Moderation: interaction effect } & \multirow[b]{2}{*}{$\mathrm{b}$} & \multicolumn{3}{|c|}{ Mediation: indirect effect } \\
\hline & $\mathrm{b}$ & $\mathrm{SE}$ & $\mathrm{p}$ & $\% R^{2}$ & & SE & $\mathrm{p}$ & $\overline{\mathrm{MC} \mathrm{CI}}$ \\
\hline Frn & -0.06 & 0.10 & 0.72 & 08 & 0.04 & 0.02 & $<0.05 *$ & $.01-.08$ \\
\hline Fms & 0.03 & 0.09 & 0.76 & 08 & 0.05 & 0.02 & $<0.01 *$ & $.02-.08$ \\
\hline Fmc & -0.07 & 0.09 & 0.72 & 09 & 0.12 & 0.02 & $<0.001 *$ & $.08-.17$ \\
\hline Pst & 0.04 & 0.08 & 0.72 & 13 & 0.11 & 0.02 & $<0.001 *$ & $.07-.17$ \\
\hline $\mathrm{Ngt}$ & 0.20 & 0.09 & 0.24 & 16 & 0.10 & 0.03 & $<0.001 *$ & $.05-.15$ \\
\hline Brd & 0.07 & 0.09 & 0.72 & 13 & 0.06 & 0.02 & $<0.05^{*}$ & $.02-.11$ \\
\hline Rfl & 0.08 & 0.09 & 0.72 & 07 & 0.01 & 0.01 & 0.41 & $-.01-.04$ \\
\hline Dst & -0.17 & 0.09 & 0.24 & 06 & 0.05 & 0.01 & $<0.001 *$ & $.02-.08$ \\
\hline Agg & 0.03 & 0.08 & 0.76 & 08 & 0.06 & 0.02 & $<0.001 *$ & $.03-.10$ \\
\hline Exp & 0.05 & 0.08 & 0.72 & 04 & -0.00 & 0.01 & 0.59 & $-.02-.01$ \\
\hline D14 & -0.06 & 0.08 & 0.72 & 23 & 0.16 & 0.03 & $<0.001 *$ & $.09-.23$ \\
\hline
\end{tabular}

Note. $\mathrm{b}=$ unstandardized regression (slope) coefficient, $\mathrm{SE}=$ standard error, $\mathrm{p}=\mathrm{p}$-value, $\% \mathrm{R}^{2}=$ percentage variance explained. MC CI = Monetcarlo confidence interval. Frn = friendship support; Fms = family support; Fmc = family cohesion; Pst = positive self-esteem; $\mathrm{Ngt}=$ negative self-esteem; Brd = brooding; Rfl = reflection; Dst = distress tolerance; Agg = aggression; Exp = expressive suppression; D14 = age-14 distress. The belonging results that are not corrected for gender can be found in Supplement II.

\section{Direct, moderation and mediation effects for multiple RF models}

The theoretical models that demonstrate multiple moderator and mediator models are depicted in Figure 2.

Direct effects. When splitting the group in CA+ and CA-, the findings diverged notably. In the CA+ group six RFs (Table 4 middle panel), and in the CA- group two RFs had a significantly negative effect on age-17 distress (Table 4 right panel; see also Supplement V for variance inflation factors). In the overall sample five RFs were significantly negatively associated with age-17 distress, namely: family cohesion, positive self-esteem, negative selfesteem, brooding and aggression (Table 4 left panel). Yet, of those five RFs only brooding had a significantly negative effect in both the $\mathrm{CA}+$ and the CA-group. Positive self-esteem had a significantly negative effect in the $\mathrm{CA}+$ and a marginally negative effect in the CA-group ( $p$ $=0.07)$.

Table 4

Multiple main effects of the RFs with correction for gender, for the whole sample, CA+ and the CAgroup

\begin{tabular}{|c|c|c|c|c|c|c|c|c|c|c|}
\hline & \multicolumn{4}{|c|}{ Whole sample } & \multicolumn{3}{|c|}{$\mathrm{CA}+$} & \multicolumn{3}{|c|}{ CA- } \\
\hline & $\mathrm{b}$ & $b^{*}$ & $\mathrm{SE}$ & $\mathrm{p}$ & $\mathrm{b}$ & $\mathrm{SE}$ & $\mathrm{p}$ & $\mathrm{b}$ & $\mathrm{SE}$ & $\mathrm{p}$ \\
\hline CA & 0.25 & - & .07 & $<0.001 *$ & - & - & - & - & - & - \\
\hline Frn & -0.08 & -0.07 & .05 & 0.12 & -0.15 & .07 & $<0.05^{*}$ & 0.03 & .08 & 0.66 \\
\hline Fms & -0.04 & -0.05 & .06 & 0.50 & 0.01 & .08 & 0.86 & -0.15 & .08 & 0.06 \\
\hline Fmc & -0.12 & -0.09 & .06 & $<0.05^{*}$ & -0.17 & .08 & $<0.05^{*}$ & 0.03 & .08 & 0.76 \\
\hline Pst & -0.17 & -0.16 & .05 & $<0.001 *$ & -0.18 & .07 & $<0.01 *$ & -0.14 & .08 & 0.07 \\
\hline $\mathrm{Ngt}$ & -0.20 & -0.20 & .07 & $<0.01 *$ & -0.06 & .09 & 0.54 & -0.40 & .10 & $<0.001 *$ \\
\hline Brd & -0.18 & -0.18 & .06 & $<0.01 *$ & -0.17 & .09 & $<0.05^{*}$ & -0.18 & .09 & $<0.05 *$ \\
\hline Rfl & 0.01 & 0.00 & .06 & 0.93 & 0.02 & .08 & 0.82 & -0.05 & .08 & 0.50 \\
\hline Dst & -0.07 & -0.05 & .04 & 0.11 & -0.12 & .06 & $<0.05 *$ & 0.03 & .06 & 0.68 \\
\hline Agg & -0.12 & -0.11 & .04 & $<0.01 *$ & -0.14 & .05 & $<0.01 *$ & -0.07 & .06 & 0.26 \\
\hline Exp & 0.05 & 0.06 & .04 & 0.16 & 0.05 & .05 & 0.27 & 0.05 & 05 & 0.39 \\
\hline gender & -0.11 & -0.11 & .08 & 0.15 & -0.08 & .11 & 0.45 & -0.15 & .11 & 0.20 \\
\hline
\end{tabular}


Note. $\mathrm{b}=$ unstandardized regression (slope) coefficient, $\mathrm{b}^{*}=$ unstandardized regression (slope) coefficient corrected for CA; $\mathrm{SE}=$ standard error, $\mathrm{p}=\mathrm{p}$-value, $\% \mathrm{R}^{2}=$ percentage variance explained. Of note, $\mathrm{SE}, \mathrm{p}$ and $\% \mathrm{R}^{2}$ belong to the effects that are not corrected for $\mathrm{CA}$. $\mathrm{CA}=$ childhood adversity, $\mathrm{CA}+=$ adolescents with $\mathrm{CA}, \mathrm{CA}-=$ adolescents without $\mathrm{CA}$; Frn = friendship support; Fms = family support; Fmc $=$ family cohesion; Pst $=$ positive self-esteem; Ngt $=$ negative self-esteem; Brd $=$ brooding; $\mathrm{Rfl}=$ reflection; Dst = distress tolerance; Agg = aggression; Exp = expressive suppression. The belonging results that are not corrected for gender can be found in Supplement II.

Moderation. CA was as expected positively associated with distress. Negative selfesteem revealed a significant moderation effect (see Figure 3; or Supplement VI for details). Yet, negative self-esteem had no relationship with age-17 distress in the CA+ group, and a significantly negative relationship with age-17 distress in the CA-group (see Table 4). Hence, negative self-esteem had the opposite effect as an RF. Friendship support, family cohesion and distress tolerance had a negative relationship with age-17 distress in the CA+ group, and no relationship with age-17 distress in the CA- group. Yet, their moderation effects did not reach significance (friendship support $p=0.07$, family cohesion $p=0.09$, and distress tolerance $p=$ 0.09).

Mediation. We first computed the multiple mediation models and examined which RFs had a negative relationship with both CA (a-path) and distress (b-path; when controlling for the b-paths of all other RFs and the CA effect), as only those can function as mediating RFs. Eight RFs qualified (of note, we also included RFs with nonsignificant paths in the cumulative mediation effect as long as the paths had the correct slope sign/directionality; see Figure 3). Expressive suppression and reflection did not qualify as they did not have a negative relationship with age-17 distress. Of the eight potential RF mediators three functioned as significant partial mediators, namely: positive self-esteem, negative self-esteem, and aggression. Brooding revealed a marginal mediation effect $(p=0.06)$. All eight RFs together had a significant cumulative mediation effect.

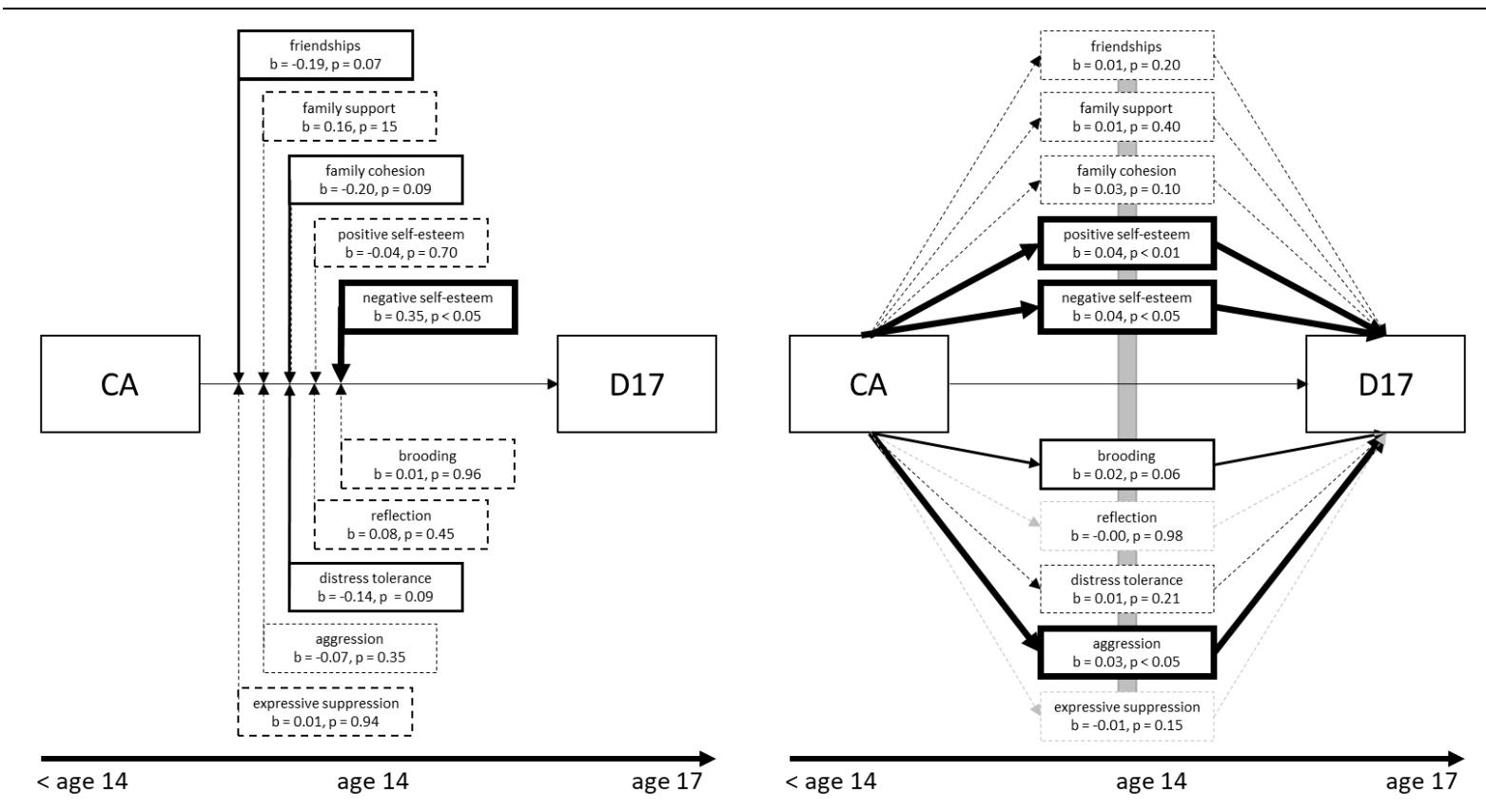

Figure 3. $\mathrm{CA}=$ childhood adversity, D17 = distress at age 17. The left panel depicts a multiple moderator model and the right panel the multiple mediator model, both corrected for gender. Bold, solid lines = significant effects; thin, solid lines = marginal effects $(0.05 \geq p<0.10)$; black, dashed lines = non-significant effects. Grey, dashed lines = nonsignificant effects of those variables that are not included in the cumulative mediation effect, for reasons detailed in the main text. The grey bar in the mediation model indicates that the RFs are allowed to correlate with each other. 


\section{DISCUSSION}

We aimed to shed light on the effects that best describe how RFs reduce mental health problems after CA. To this end we looked at direct, moderation and mediation effects. We first investigated models separately for the ten RFs, so called single RF models. We found that eight out of 10 RFs had a significantly negative direct effect on age-17 distress in both the CA+ and the CA- group, and thus also in the overall sample. While no RF revealed a significant moderation effect, as many as eight of the ten RFs revealed a significant mediation effect. Next, we tested models including all ten RFs, so called multiple RF models, to safeguard overestimating single RF effects and to take the ecological validity sufficiently into account. This time, four RFs had a significantly negative direct effect in the overall sample. Yet, of those four, only brooding revealed a significant effect in both groups. Positive self-esteem had a significant effect in the CA+ group, but only a marginal effect in the CA- group. The moderation effects of friendship support, family cohesion and distress tolerance did not reach significance, despite that all three RFs had a significantly negative relationship with age-17 distress in the $\mathrm{CA}+$ group, and no effect in the CA- group. Positive self-esteem, negative selfesteem and aggression functioned as significant mediators. Brooding revealed a borderline mediation effect. All in all, our findings provide predominant support for RFs to function as mediators rather than as moderators, regardless of investigating single or multiple RF models. Below, I shall first embed the moderation and then the mediation results within the wider literature.

Previous literature has demonstrated that distress tolerance ${ }^{38}$ and family climate (similar to family cohesion) ${ }^{39}$ moderate the relationship between CA and subsequent distress, in single RF models (see also Table 5). In other words, intra-personal distress acceptance and inter-personal cohesion with the next of kin were found to reduce subsequent distress particularly in those adolescents with a history of CA. We did not find corresponding effects in single RF models. Moreover, in our multiple RF model, both the moderation effect of distress tolerance and of family cohesion only approached significance. Whether such marginal effects should or should not be interpreted, has been debated by scientists since decades. ${ }^{40}$ Neyman and Pearson $(1928)^{41}$, two key developers of statistical hypothesis testing, stated that "it is doubtful whether the knowledge that $P$ was really .03 (or .06) rather than $.05 \ldots$ would in fact ever modify our judgment" (p. 201) ${ }^{41}$ and explain that "the tests should only be regarded as tools which must be used with discretion and understanding, and not as instruments which in themselves give the final verdict" (p. 232). ${ }^{41}$ To err on the side of caution, we will here not interpret the marginal moderation effects and will thus not draw conclusions regarding differences between adolescents with and without CA. However, we do interpret the fact that distress tolerance and family cohesion reduced subsequent distress significantly in the group of adolescents with CA, even after correcting for the other RFs. In sum, given our fairly inconclusive moderation findings, our results diverge slightly from the reviewed findings, which indicated preliminary support for the moderation effects of distress tolerance and family cohesion. Yet, our findings do to some degree reveal a similar conclusion. That is, when taking an ecological stance - taking into account the existence of multiple potential RFs - distress tolerance and family cohesion seemed to be important factors for decreasing subsequent distress in adolescents with prior exposure to adversity. Whether the protective value of distress tolerance and family cohesion differs eventually significantly between groups of adolescents with and without CA (or not) needs to be re-tested in future research.

For social support the literature is not conclusive, including some studies that do find, ${ }^{39}$ and others that do not find support for a moderation effect. ${ }^{42} \mathrm{We}$ found no moderation effect in the single RF model, and only a marginal effect in the multiple RF model. Yet, as for distress tolerance and family cohesion, friendship support reduced subsequent distress only 
significantly in the group of adolescents with CA, and not in the group without CA. As before, while we interpret the significant promotive effect of friendship support in the CA+ group, we refrain from drawing conclusions regarding the marginal difference between the two groups (i.e. the marginal moderation effect). Based on the results of the reviewed studies, one may wonder whether friendship support reduces internalizing symptoms particularly after the exposure to family-related CA (e.g. parental psychopathology ${ }^{39}$ ), but less so for CA that has taken place outside the family (e.g. rocket attacks ${ }^{42}$ or bullying ${ }^{43}$ ). Hence, it may be the case that friendships are particularly beneficial in maintaining or increasing mental health after exposure to family-related CA. This would make sense, as a disadvantageous family environment may in itself be less supportive and sometimes even hazardous, in which case adolescents may benefit from seeking support outside of the family. Such a conjecture would align with cross-domain support matching. ${ }^{44}$ Yet, some research suggests that cross-domain support matching has not proven to be effective. ${ }^{44}$

While the literature did reveal some support for RF moderation effects, our research revealed at best very weak, marginal interaction effects, rendering our moderation results inconclusive. One possible account may come from our sample size, which was smaller than for example the sample used in prior literature for family cohesion and social support $(N>$ 1500). Another account may come from the fact that our CA variable was binary, and therefore potentially less sensitive than an index reflecting CA severity or frequency. The binary CA variable may have, in addition to the restricted sample size, limited the explanatory power. A third explanation may, as above explained for friendship support, be that RF moderation effects depend on the specific type of CA. It seems unlikely that our mental health outcome variable has contributed to the divergence from previous findings, as the reported previous literature either used anxiety or depression symptoms as outcome variable, which are both captured in our transdiagnostic distress index.

Table 5

Moderation and Mediation Effects of Single and Multiple RF Models, Found in the Literature

\begin{tabular}{|c|c|c|c|c|c|c|c|c|c|c|}
\hline \multirow{2}{*}{$\begin{array}{l}\text { Effect } \\
\text { Moderation }\end{array}$} & Frn & Fms & Fmc & Pst & $\mathrm{Ngt}$ & Brd & Rfl & Dst & Agg & Exp \\
\hline & $\sqrt{ } 39$ & $\begin{array}{l}{ }^{42 * 1} \\
2^{*} * 14\end{array}$ & $\sqrt{39}$ & $\sqrt{\checkmark^{45 * 3}}$ & $\sqrt{45 * 3}$ & $x^{46^{* 4}}$ & $x^{46^{* 4}}$ & $\sqrt{38}$ & & $x^{46 * 4}$ \\
\hline \multirow{2}{*}{ Mediation } & $\begin{array}{l}x^{2+1} \\
x^{43}\end{array}$ & $\begin{array}{l}\mathbf{x}^{42-1,43} \\
\sqrt{43}\end{array}$ & $\sqrt{ } 47 * 2$ & & & $\sqrt{ }^{46 * 4}$ & $\checkmark^{46 * 4}$ & & $\checkmark^{4}$ & $\sqrt{46 * 4}$ \\
\hline & & & $x^{47 * 2}$ & & & $x^{48}$ & $x^{48}$ & & $\mathbf{x}^{49}$ & \\
\hline
\end{tabular}

Note. Frn = friendship support; Fms = family support; Fmc = family cohesion; Pst = positive self-esteem; Ngt $=$ negative selfesteem; Brd = brooding; Rfl = reflection; Dst = distress tolerance; Agg = aggression; Exp = expressive suppression. $\checkmark=$ this effect was tested and found; $\boldsymbol{x}=$ this effect was tested but not found; *effects found in multiple RF models, taking into account the following RFs: ${ }^{1}=$ friend support, family support, school support; ${ }^{2}=$ family cohesion, communication with the mother, communication with the father; ${ }^{3}=$ self-esteem, academic grades, positive parenting $;{ }^{4}=$ rumination, expressive suppression, cognitive reappraisal (although the respective moderation effect was only corrected for the main effects, not the interaction effects of the other RFs). Importantly, a detailed synthesis and narrative for all the findings that are contained in Table 5, can be found in the original systematic review from which this information is taken, see $^{6}$.

With regard to mediation effects, previous literature has demonstrated that aggression ${ }^{49}$ and brooding ${ }^{46}$ function as intermediate variables between CA and subsequent mental health problems. Yet, the literature is not fully conclusive and contains some findings that do not support the suggested mediation effects (see e.g. ${ }^{49}$ for aggression and ${ }^{48}$ for brooding). While the literature revealed a mediation effect for aggression in a single RF model, our research found support in single as well as multiple RF models. Moreover, the literature revealed a mediation effect for brooding in a multiple RF model, whereas we found predominant support for brooding in the single RF model and only marginal support in the multiple RF model. Interestingly, while self-esteem has previously been shown to function as a moderator, ${ }^{45}$ our results rather indicate that positive and negative self-esteem are important mediators for the 
relationship between CA and age-17 distress. Furthermore, we found that the cumulative RF mediation effect was particularly robust and strong. Overall, the probably most notable finding of our study is that while all revealed moderation effects were weak and marginal, most mediation effects were strong and robust. This could for example be related to power. As explained above, our binary CA variable had a limited explanatory power, which may have predominantly disadvantaged the highly power sensitive moderation effects (see our power calculations in Supplement IV). However, the fact that we mainly found support for mediation than for moderation could also simply be the correct result, given the statistical nature of the two different effects. That is, RF moderators need to have a stronger effect on distress in the $\mathrm{CA}+$ than in the CA- group, whereas RF mediators can have a comparably strong effect on distress in the $\mathrm{CA}+$ and the $\mathrm{CA}$ - groups.

Besides the discussion on the relevance of CA-RF matching, a highly interesting consideration is how transdiagnostic RF effects are. For many of the most robust RFs there seems to be some preliminary transdiagnostic evidence. For instance, for ruminative brooding there is a broad and solid literature that supports its relationship with depression related disorders, anxiety related disorders and obsessive compulsive disorder, as well as some evidence for its association with bulimia and alcohol abuse. ${ }^{50}$ There is preliminary but good support for self-esteem, particularly for its effects on internalizing related disorders. ${ }^{51}$ Moreover, anger (i.e. a form of aggression) was found in conjunction with other emotion regulation skills to function as transdiagnostic effect on internalizing and externalizing symptoms. ${ }^{52}$ Similarly, social support, ${ }^{53}$ family functioning ${ }^{54}$ and distress tolerance ${ }^{53}$ have been suggested to function as transdiagnostic effects on mental distress symptoms. Yet, knowledge on anger or aggression, social support, family functioning and distress tolerance seems so far limited, or seems at least to lack synopsis, and more meta-synthesis is needed. Unsurprisingly, self-esteem, brooding and emotion-regulation have already been found to be successful intervention targets, ${ }^{55-57}$ particularly for interventions aimed at reducing internalizing disorders and/or increasing mental well-being.

Our findings suggest that improving levels of many of the tested RFs in early adolescence may improve mental health during later adolescence and young adulthood. However, in which way does this knowledge inform translational research or (upon replication) clinical settings? Technically both moderation and mediation effects may lend themselves well for prevention and treatment. Speculatively, one could assume that mediation effects may be particularly advantageous for prevention, as they require a negative impact of the CA exposure on the RF as well as an increased risk of mental distress after CA. Hence, if prevention would start to enhance the RF early on, the deleterious effect of CA on the RF could be disrupted, and the enhanced RF could in turn foster or increase mental health at an early stage, ideally before the onset of mental health problems. For moderation effects, such a consideration is less relevant as CA does not need to directly relate to the RF. Important is however, that the RF moderator reduces subsequent distress more in adolescents with than without exposure to CA. Such an effect is therefore more easily detected when the RF has no effect on distress in the group of adolescents without CA. Yet, RFs that are not also protective for adolescents without CA should not be targeted in interventions aimed at both individuals with and without CA. Moreover, such RFs should ideally also not be targeted when a CA history is unknown and cannot be assessed, as it is sometimes the case in school or community settings. When the aim is to prioritise interventions aimed at all adolescents regardless of a history of CA, one should focus on those RFs that are successful in reducing distress significantly in both the group of adolescents with and without CA. Based on our findings one would need to enhance low brooding and potentially positive self-esteem. Importantly, our findings only suggest potentially relevant factors for interventions, but we first need evidence of how effective those 
RFs actually are, when being targeted in interventions, before we can be certain about their effects.

A related and still largely unanswered question is whether it is more efficient to target and enhance RFs that adolescents have already acquired, or to target RFs that adolescents have not yet acquired well. Theoretically both seems plausible. RF skills or resources that are already present may be particularly efficient targets, as they may be relatively easy to be detected and capitalized on, but may induce limited change. Therefore, one may wonder whether those RFs may be particularly helpful for acute but limited improvement in mental health. RF skills and resources that are only immaturely developed may be particularly effective targets, as they may be difficult and time-consuming to acquire, but may induce more change once acquired. Therefore, one may wonder whether those RFs may be particularly helpful for slow but substantial improvement in mental health. According to Ellis and colleagues (2017) ${ }^{58}$ interventions that focus on disadvantaged skills have not yet proven to be helpful and have particularly established how hard it is to induce effective skill-set changes following CA. Another important consideration when choosing RF targets, also discussed by Ellis and colleagues (2017), ${ }^{58}$ is that it may be dangerous to "de-claw the cat" (Ellis et al., 2017 $7^{58}$; p. 566). In other words, it may be safer to improve already existing skills, rather than trying to induce new or revert skills, particularly when those are likely to disadvantage the adolescents in some contexts. For instance, while in general it may be advantageous to improve distress tolerance skills in adolescents (i.e. reduce distress sensitivity), it may for some adolescents be disadvantageous or even dangerous. Taking adolescents in violent home environments as an example, it seems logical that low distress tolerance, as in being sensitive and alert when situations generate friction, may actually be advantageous. Therefore, it seems highly important to (a) be aware of the underlying type of the protective RF effect, so that appropriate expectations can be set, and to (b) be aware for which particular group (or adolescent) the RF target at hand is suitable. Both requirements for choosing RF targets for translational research clearly need further scientific evaluation.

Our research is of course not without limitations. Besides sampling restrictions, such as an above-average prosperity, ${ }^{7}$ the study also had limitations regarding the measurement. For example, some RFs were based on subscales of the same questionnaire (which may result in higher correlations due to equivalent answer options), and not all questionnaires were developed for the specific RFs (e.g. expressive suppression). For future research it would be advisable to use scales particularly set out for the specific RFs, as this is likely to increase the psychometric quality. Along those lines, the dichotomous CA variable which we have used may be restricted in range, which may, as explained above, have limited the explanatory power. Future research may besides CA exposure also want to assess the severity and frequency of the exposure. ${ }^{59}$ Moreover, in addition to a retrospective assessment, a prospective assessment would be ideal. Yet, an advantage of our CA variable is that CA was assessed by caregiver report, whereas most RFs (eight out of 10) and distress were assessed via adolescent self-report. Therefore, the adversity variable cannot be directly biased by the adolescents' mood state. Another potential limitation may come from the missing data treatment. Here we used predictive mean matching (for ordered categorical items) and logistic algorithms (for binary items) to estimate missing values. We imputed the RFs and distress on the item level and then ran factor analyses on the imputed items, as the lower the level on which the imputation is performed, the more information is available and thus bias is less likely. As we did not perform the imputations on the latent (factor score) level, we could not include interactions between CA and the RFs in the imputation analysis. Yet, research has shown that moderation analyses based on imputed data are generally more precise when interaction terms of the variables are included in the imputation model, as well. ${ }^{60}$ To provide the reader with as much information as possible, we also computed all analyses based on non-imputed data. However, given the item-level 
imputation approach, which we have chosen to prioritize imputation precision, we cannot disentangle whether differences in moderation results between imputed and non-imputed data are the result of a potentially insufficiently complex imputation model or because of selection bias in the non-imputed sample. Last but not least, it is important to remind the reader that all results are solely derived from between-person analyses, indicating group level effects, and are thus likely to not directly translate to the individual (idiographic) level.

In sum, we showed that improving levels of many of the tested RFs in early adolescence may improve mental health during later adolescence and young adulthood. Specifically, enhancing self-esteem, low brooding, and low aggression may disrupt the deleterious effect of $\mathrm{CA}$ and thereby improve or stabilize mental health. Our findings further suggest that for settings in which we cannot assess adversity, one may want to focus on enhancing low brooding, as low brooding seems to reduce subsequent mental health problems regardless of a history of CA. Translational research may be advised to match the purpose of the studied intervention closely with the specific type of the RF effect of interest. 


\section{REFERENCES}

1. Bonanno, G. A., Westphal, M. \& Mancini, A. D. Resilience to Loss and Potential Trauma. Annu. Rev. Clin. Psychol. 7, 511-535 (2011).

2. Kessler, R. C. et al. Childhood adversities and adult psychopathology in the WHO World Mental Health Surveys. Br. J. Psychiatry 197, 378-385 (2010).

3. Greif Green, J. et al. Childhood adversities and adult psychopathology in the National Comorbidity Survey Replication (NCS-R) I: Associations with first onset of DSM-IV disorders. Arch. Gen. Psychiatry 67, 113 (2010).

4. McLaughlin, K. A. et al. Childhood Adversities and First Onset of Psychiatric Disorders in a National Sample of US Adolescents. Arch. Gen. Psychiatry 69, 11511160 (2012).

5. McLaughlin, K. A. Future Directions in Childhood Adversity and Youth Psychopathology. J. Clin. Child Adolesc. Psychol. 45, 361-382 (2016).

6. Fritz, J., de Graaff, A.-M., Caisley, H., van Harmelen, A.-L. \& Wilkinson, P. O. A Systematic Review of Amenable Resilience Factors that Moderate and/or Mediate the Relationship between Childhood Adversity and Mental Health in Young People.

Front. Psychiatry 9, 230 (2018).

7. Goodyer, I. M., Croudace, T., Dunn, V., Herbert, J. \& Jones, P. B. Cohort Profile: Risk patterns and processes for psychopathology emerging during adolescence: the ROOTS project. Int. J. Epidemiol. 39, 361-369 (2010).

8. Fritz, J. et al. Unravelling the complex nature of resilience factors and their changes between early and later adolescence. BMC Med. 17, 203 (2019).

9. Fritz, J., Stochl, J., Goodyer, I. M., van Harmelen, A.-L. \& Wilkinson, P. O.

Embracing the positive: An examination of how well resilience factors at age 14 can predict distress at age 17. Transl. Psychiatry 10, 272 (2020).

10. Chmura Kraemer, H., Stice, E., Kazdin, A., Offord, D. \& Kupfer, D. How Do Risk Factors Work Together? Mediators, Moderators, and Independent, Overlapping, and Proxy Risk Factors. Am. J. Psychiatry 158, 848-856 (2001).

11. Holmbeck, G. N. Toward Terminological, Conceptual, and Statistical Clarity in the Study of Mediators and Moderators: Examples From the Child-Clinical and Pediatric Psychology Literatures. J. Consult. Clin. Psychol. 65, 599-610 (1997).

12. Rose, B. M., Holmbeck, G. N., Millstein Coakley, R. \& Franks, E. A. Mediator and Moderator Effects in Developmental and Behavioral Pediatric Research. Dev. Behav. Pediatr. 25, 58-67 (2004).

13. Dunn, V. J. et al. Profiles of family-focused adverse experiences through childhood and early adolescence: The ROOTS project a community investigation of adolescent mental health. BMC Psychiatry 11, 109 (2011).

14. Fritz, J., Fried, E. I., Goodyer, I. M., Wilkinson, P. O. \& van Harmelen, A.-L. A Network Model of Resilience Factors for Adolescents with and without Exposure to Childhood Adversity. Sci. Rep. 8, 15774 (2018).

15. Goodyer, I. M., Wright, C. \& Altham, P. M. E. Recent friendships in anxious and depressed school age children. Psychol. Med. 19, 165-174 (1989).

16. Epstein, N. B., Baldwin, L. M. \& Bishop, D. S. The McMaster Family Assessment Device. J. Marital Fam. Ther. 9, 171-180 (1983). 
17. Rosenberg, M. Society and the Adolescent Self-Image. (Princeton, NJ: Princeton University Press, 1965).

18. Treynor, W., Gonzalez, R. \& Nolen-Hoeksema, S. Rumination reconsidered: A psychometric analysis. Cognit. Ther. Res. 27, 247-259 (2003).

19. Burwell, R. A. \& Shirk, S. R. Subtypes of rumination in adolescence: Associations between brooding, reflection, depressive symptoms, and coping. J. Clin. Child Adolesc. Psychol. 36, 56-65 (2007).

20. Bould, H., Joinson, C., Sterne, J. \& Araya, R. The Emotionality Activity Sociability Temperament Survey: Factor analysis and temporal stability in a longitudinal cohort. Pers. Individ. Dif. 54, 628-633 (2013).

21. Goodyer, I. M. et al. Improving mood with psychoanalytic and cognitive therapies (IMPACT): a pragmatic effectiveness superiority trial to investigate whether specialised psychological treatment reduces the risk for relapse in adolescents with moderate to severe unipolar dep... Trials 12, 175 (2011).

22. American Psychiatric Association. Diagnostic and statistical manual of mental disorders (4th ed., text rev.). (Washington, DC: Author, 2000).

23. Poythress, N. G. et al. Internal Consistency Reliability of the Self-Report Antisocial Process Screening Device. Assessment 13, 107-113 (2006).

24. Wood, A., Kroll, L., Moore, A. \& Harrington, R. Properties of the Mood and Feelings Questionnaire in Adolescent Psychiatric Outpatients: A Research Note. J. Child Psychol. Psychiatry 36, 327-334 (1995).

25. Reynolds, C. R. \& Richmond, B. O. What I Think and Feel: A Revised Measure of Children's Manifest Anxiety. J. Abnorm. Child Psychol. 6, 271-280 (1978).

26. St Clair, M. C. et al. Characterising the latent structure and organisation of selfreported thoughts, feelings and behaviours in adolescents and young adults. PLoS One 12, e0175381 (2017).

27. Brodbeck, J., Abbott, R. A., Goodyer, I. M. \& Croudace, T. J. General and specific components of depression and anxiety in an adolescent population. BMC Psychiatry 11, 191 (2011).

28. Stochl, J. et al. Mood, anxiety and psychotic phenomena measure a common psychopathological factor. Psychol. Med. 45, 1483-1493 (2015).

29. van Buuren, S. \& Groothuis-Oudshoorn, K. mice: Multivariate Imputation by Chained Equations in R. J. Stat. Softw. 45, 1-67 (2011).

30. Wu, H. \& Estabrook, R. Identification of Confirmatory Factor Analysis Models of Different Levels of Invariance for Ordered Categorical Outcomes. Psychometrika 81, 1014-1045 (2016).

31. MacKinnon, D. P., Fairchild, A. J. \& Fritz, M. S. Mediation Analysis. Annu. Rev. Psychol. 58, 593-614 (2007).

32. MacKinnon, D. P., Lockwood, C. M. \& Williams, J. Confidence Limits for the Indirect Effect: Distribution of the Product and Resampling Methods. Multivariate Behav. Res. 39, 99 (2004).

33. Rosseel, Y. lavaan: An R package for structural equation modeling. J. Stat. Softw. 48, 1-36. Retrieved from http://www.jstatsoft.org/v48/ (2012).

34. R Core Team. R: A language and environment for statistical computing. R Found. Stat. 
Comput. Vienna, Austria. Retrieved from https://www.R-project.org/ (2018).

35. Muthén, L. K. \& Muthén, B. O. Mplus User's Guide. Eights Edition. (Muthén \& Muthén, 2017).

36. Morgan, M. \& Chinn, S. ACORN group, social class, and child health. J. Epidemiol. Community Health 37, 196-203 (1983).

37. Kaufman, J. et al. Schedule for Affective Disorders and Schizophrenia for School-Age Children-Present and Lifetime Version (K-SADS-PL): Initial reliability and validity data. J. Am. Acad. Child Adolesc. Psychiatry 36, 980-988 (1997).

38. Banducci, A. N., Lejuez, C. W., Dougherty, L. R. \& MacPherson, L. A Prospective Examination of the Relations Between Emotional Abuse and Anxiety: Moderation by Distress Tolerance. Prev. Sci. 18, 20-30 (2017).

39. Klasen, F. et al. Risk and protective factors for the development of depressive symptoms in children and adolescents: results of the longitudinal BELLA study. Eur. Child Adolesc. Psychiatry 24, 695-703 (2015).

40. Greenland, S. et al. Statistical tests, $\mathrm{P}$ values, confidence intervals, and power: a guide to misinterpretations. Eur. J. Epidemiol. 31, 337-350 (2016).

41. Neyman, J. \& Pearson, E. S. On the Use and Interpretation of Certain Test Criteria for Purposes of Statistical Inference: Part I. Biometrika 20A, 175-240 (1928).

42. Shahar, G. \& Henrich, C. C. Perceived Family Social Support Buffers Against the Effects of Exposure to Rocket Attacks on Adolescent Depression, Aggression, and Severe Violence. J. Fam. Psychol. 30, 163-168 (2015).

43. van Harmelen, A.-L. et al. Friendships and Family Support Reduce Subsequent Depressive Symptoms in At-Risk Adolescents. PLoS One 11, e0153715 (2016).

44. Gore, S. \& Aseltine Jr., R. H. Protective Processes in Adolescence: Matching Stressors with Social Resources. Am. J. Community Psychol. 23, 301-327 (1995).

45. Dubow, E. F. et al. Exposure to Political Conflict and Violence and Posttraumatic Stress in Middle East Youth: Protective Factors. J. Clin. Child Adolesc. Psychol. 41, 402-416 (2012).

46. Boyes, M. E., Hasking, P. A. \& Martin, G. Adverse Life Experience and Psychological Distress in Adolescence: Moderating and Mediating Effects of Emotion Regulation and Rumination. Stress Heal. 32, 402-410 (2015).

47. Finan, L. J., Schulz, J., Gordon, M. S. \& McCauley Ohannessian, C. Parental problem drinking and adolescent externalizing behaviors: The mediating role of family functioning. J. Adolesc. 43, 100-110 (2015).

48. Gaté, M. A. et al. Maternal Parenting Behaviors and Adolescent Depression: The Mediating Role of Rumination. J. Clin. Child Adolesc. Psychol. 42, 348-357 (2013).

49. You, S. \& Lim, S. A. Development pathways from abusive parenting to delinquency: The mediating role of depression and aggression. Child Abuse Negl. 46, 152-162 (2015).

50. Wahl, K. et al. Is repetitive negative thinking a transdiagnostic process? A comparison of key processes of RNT in depression, generalized anxiety disorder, obsessivecompulsive disorder, and community controls. J. Behav. Ther. Exp. Psychiatry 64, 4553 (2019).

51. Keane, L. \& Loades, M. Review: Low self-esteem and internalizing disorders in young 
people - a systematic review. Child Adolesc. Ment. Health 22, 4-15 (2017).

52. McLaughlin, K. A., Hatzenbuehler, M. L., Mennin, D. S. \& Nolen-Hoeksema, S. Emotion dysregulation and adolescent psychopathology: A prospective study. Behav. Res. Ther. 49, 544-554 (2011).

53. Cohen, J. R., Danielson, C. K., Adams, Z. W. \& Ruggiero, K. J. Distress Tolerance and Social Support in Adolescence: Predicting Risk for Internalizing and Externalizing Symptoms Following a Natural Disaster. J. Psychopathol. Behav. Assess. 38, 538-546 (2016).

54. Santesteban-Echarri, O. et al. Family functioning in youth at-risk for serious mental illness. Compr. Psychiatry 87, 17-24 (2018).

55. Millar, S. L. \& Donnelly, M. Promoting mental wellbeing: developing a theoretically and empirically sound complex intervention. J. Public Health (Bangkok). 36, 275-284 (2013).

56. Watkins, E. Psychological treatment of depressive rumination. Curr. Opin. Psychol. 4, 32-36 (2015).

57. Kovacs, M. et al. Contextual emotion-regulation therapy for childhood depression: Description and pilot testing of a new intervention. J. Am. Acad. Child Adolesc. Psychiatry 45, 892-903 (2006).

58. Ellis, B. J., Bianchi, J. M., Griskevicius, V. \& Frankenhuis, W. E. Beyond Risk and Protective Factors: An Adaptation-Based Approach to Resilience. Perspect. Psychol. Sci. 12, 561-587 (2017).

59. Schlechter, P., Fritz, J. \& Wilkinson, P. O. Exploring Ways of Measuring Childhood Adversity: A Comparison of Presence vs. Absence, Severity and Frequency Indicators. Open Sci. Framew. Prepr. (2019). doi:https://osf.io/zf8r9/

60. White, I. R., Royston, P. \& Wood, A. M. Multiple imputation using chained equations: Issues and guidance for practice. Stat. Med. 30, 377-399 (2011).

61. Messer, S. C., Angold, A. \& Costello, E. J. Development of a Short Questionnaire for Use in Epidemiological Studies of Depression in Children and Adolescents: Factor Composition and Structure across Development. Int. J. Methods Psychiatr. Res. 5, 251-262 (1995).

62. Fritz, M. S. \& MacKinnon, D. P. A graphical representation of the mediated effect. Behav. Res. Methods 40, 55-60 (2008).

63. Fritz, M. S. \& MacKinnon, D. P. med_plots.R. Retrieved June 21, 2018 from Psychonomic Society Web Archive: www.psychonomic.org/archive. www.psychonomic.org/archive (2008).

64. Fox, J. \& Weisberg, S. An $\{R\}$ Companion to Applied Regression (Second Edition). (Thousand Oaks: Sage., 2011).

65. Wickham, H., François, R., Henry, L. \& Müller, K. dplyr: A Grammar of Data Manipulation. R package version 0.7.7. (2018). Available at: https://cran.rproject.org/package $=$ dplyr.

66. R Core Team. foreign: Read Data Stored by 'Minitab', 'S', 'SAS', 'SPSS', 'Stata', 'Systat', 'Weka', 'dBase', .... R package version 0.8-70. (2017).

67. Grosjean, P. \& Ibanez, F. pastecs: Package for Analysis of Space-Time Ecological Series. R package version 1.3.21. (2018). Available at: https://cran.r- 
project.org $/$ package $=$ pastecs.

68. Johnson, P. E. rockchalk: Regression Estimation and Presentation. R package version 1.8.129. (2018).

69. Jorgensen, T. D., Pornprasertmanit, S., Schoemann, A. M. \& Rosseel, Y. semTools: Useful tools for structural equation modeling. R package version 0.5-1.933. (2018). Available at: https://cran.r-project.org/package=semTools.

70. Lüdecke, D. sjPlot: Data Visualization for Statistics in Social Science. R package version 2.6.2. (2018).

71. Faul, F., Erdfelder, E., Lang, A.-G. \& Buchner, A. G*Power 3: A flexible statistical power analysis program for the social, behavioral, and biomedical sciences. Behav. Res. Methods 39, 175-191 (2007).

72. Schoemann, A. M., Boulton, A. J. \& Short, S. D. Determining Power and Sample Size for Simple and Complex Mediation Models. Soc. Psychol. Personal. Sci. 8, 379-386 (2017). 


\section{SUPPLEMENTS}

\section{Supplement I}

The continuous latent distress scores used in the manuscript are based on a strongly invariant, categorical CFAs (i.e. L+T+I IM models in Table 1), to ensure the latent mean comparability between distress at age 14 and age 17. More specifically, we applied the delta parametrization, equated item loadings and item thresholds across the two time points (i.e. age 14 and 17), fixed all item intercepts to 0 , the item scales of the first time point to 1 , the latent factor mean of the first time point to 0 , and the latent factor variance of the first time point to 1 .

Table 1

(Longitudinal) Confirmatory Factor Analyses Conducted with WLSMV estimator, $n=1188$

\begin{tabular}{|c|c|c|c|c|c|c|}
\hline Model & Robust CFI & Robust TLI & Robust RMSEA & RMSEA $90 \%$ CI & SRMR & Chisq(df) \\
\hline \multicolumn{7}{|c|}{ Friendship support ${ }^{15}, 5$ items, 1 unique item covariance } \\
\hline $\mathbf{B M}$ & 0.988 & 0.969 & 0.067 & $0.043-0.093$ & 0.036 & $12.652(4)$ \\
\hline \multicolumn{7}{|c|}{ Family support ${ }^{16}, 5$ items, 1 unique item covariance } \\
\hline BM & 0.995 & 0.987 & 0.062 & $0.039-0.088$ & 0.023 & $9.285(4)$ \\
\hline \multicolumn{7}{|c|}{ Family cohesion ${ }^{16}, 7$ items, 1 unique item covariance } \\
\hline $\mathbf{B M}$ & 0.980 & 0.967 & 0.070 & $0.057-0.085$ & 0.042 & $48.773(13)$ \\
\hline \multicolumn{7}{|c|}{ Positive self-esteem ${ }^{17}, 5$ items, 1 unique item covariance } \\
\hline $\mathbf{B M}$ & 0.996 & 0.989 & 0.076 & $0.052-0.102$ & 0.016 & $9.446(4)$ \\
\hline \multicolumn{7}{|c|}{ Negative self-esteem ${ }^{17}, 5$ items, 0 unique item covariances } \\
\hline $\mathbf{B M}$ & 0.993 & 0.987 & 0.045 & $0.022-0.069$ & 0.017 & $6.542(5)$ \\
\hline \multicolumn{7}{|c|}{ Brooding ${ }^{18}, 5$ items, 0 unique item covariances } \\
\hline $\mathbf{B M}$ & 0.991 & 0.983 & 0.068 & $0.047-0.091$ & 0.029 & $14.520(5)$ \\
\hline \multicolumn{7}{|c|}{ Reflection ${ }^{18}, 5$ items, 1 unique item covariance } \\
\hline BM & 0.999 & 0.998 & 0.023 & $0.000-0.054$ & 0.018 & $3.435(4)$ \\
\hline \multicolumn{7}{|c|}{ Distress tolerance $^{20}, 5$ items, 1 unique item covariance } \\
\hline BM & 0.977 & 0.942 & 0.128 & $0.105-0.153$ & 0.051 & $36.272(4)$ \\
\hline \multicolumn{7}{|c|}{ Aggression $^{21}$, 4 items, 0 unique item covariances } \\
\hline $\mathbf{B M}$ & 0.988 & 0.965 & 0.029 & $0.000-0.071$ & 0.036 & $1.387(2)$ \\
\hline \multicolumn{7}{|c|}{ Distress ${ }^{25,61}, 41$ items, 2 unique item covariances } \\
\hline C IM 1 & 0.988 & 0.987 & 0.026 & $0.025-0.027$ & 0.043 & \\
\hline C IM 2 & 0.988 & 0.988 & 0.026 & $0.025-0.027$ & 0.043 & \\
\hline C IM 3 & 0.989 & 0.989 & 0.026 & $0.025-0.027$ & 0.043 & \\
\hline C IM 4 & 0.989 & 0.989 & 0.026 & $0.025-0.027$ & 0.043 & \\
\hline C IM 5 & 0.988 & 0.987 & 0.027 & $0.026-0.028$ & 0.045 & \\
\hline C IM 6 & 0.989 & 0.988 & 0.026 & $0.025-0.027$ & 0.044 & \\
\hline C IM 7 & 0.990 & 0.990 & 0.026 & $0.025-0.027$ & 0.044 & \\
\hline C IM 8 & 0.986 & 0.986 & 0.027 & $0.026-0.028$ & 0.047 & \\
\hline C IM 9 & 0.989 & 0.988 & 0.026 & $0.025-0.028$ & 0.045 & \\
\hline C IM 10 & 0.988 & 0.988 & 0.026 & $0.025-0.027$ & 0.045 & \\
\hline L+T+I IM 1 & 0.986 & 0.986 & 0.027 & $0.026-0.028$ & 0.042 & \\
\hline L+T+I IM 2 & 0.987 & 0.987 & 0.027 & $0.026-0.028$ & 0.042 & \\
\hline L+T+I IM 3 & 0.988 & 0.988 & 0.027 & $0.026-0.028$ & 0.042 & \\
\hline L+T+I IM 4 & 0.988 & 0.988 & 0.027 & $0.026-0.028$ & 0.043 & \\
\hline L+T+I IM 5 & 0.986 & 0.986 & 0.028 & $0.027-0.029$ & 0.045 & \\
\hline L+T+I IM 6 & 0.987 & 0.987 & 0.027 & $0.026-0.028$ & 0.043 & \\
\hline $\mathbf{L}+\mathbf{T}+\mathbf{I}$ IM 7 & 0.989 & 0.989 & 0.027 & $0.026-0.028$ & 0.043 & \\
\hline L+T+I IM 8 & 0.985 & 0.984 & 0.028 & $0.027-0.029$ & 0.046 & \\
\hline L+T+I IM 9 & 0.988 & 0.988 & 0.027 & $0.026-0.028$ & 0.044 & \\
\hline L+T+I IM 10 & 0.987 & 0.987 & 0.027 & $0.026-0.029$ & 0.044 & \\
\hline
\end{tabular}

Note. WLSMV = weighted least squares mean and variance corrected estimator; CFI = Comparative fit index; TLI = TuckerLewis index; RMSEA = Root mean square error of approximation; $\mathrm{CI}=$ Confidence interval; chisq = chi-square; $\mathrm{BM}=$ baseline model; $\mathrm{C}$ IM = configural invariance model; L+T+I IM = loadings, thresholds, and intercepts invariance model. All models were conducted with the delta parameterization. 


\section{Supplement II}

This Supplement contains all results of the main manuscript that have been corrected for gender, this time without gender correction. The below Table 2 corresponds to Table 2 in the main manuscript. The below Table 3 corresponds to Table 3 in the main manuscript. The moderation results of Table 3 are also depicted in Figure 1 and the mediation results in Figure 2. The below Table 4 corresponds to Table 4 in the main manuscript. Results for multiple moderation and mediation effects can be found in Supplement VI.

The mediation Figure 1.b2, as well as below Figure 2, are modelled using an adapted script from Fritz and MacKinnon ${ }^{62}$ (Behavior Research Methods: https://doi.org/10.3758/BRM.40.1.55) the original scripts can be found in the belonging supplement material (Fritz \& MacKinnon ${ }^{63}$, at: https://link.springer.com/article/10.3758/ BRM.40.1.55\#SupplementaryMaterial).

Table 2

Single main effects of the RFs without correction for gender, for the whole sample, the CA+ and the CA- group; corrected for multiple testing

\begin{tabular}{|c|c|c|c|c|c|c|c|c|c|c|c|c|c|}
\hline & \multicolumn{5}{|c|}{ Whole sample } & \multicolumn{4}{|c|}{ CA+ } & \multicolumn{4}{|c|}{$C A-$} \\
\hline & $\mathrm{b}$ & $\mathrm{b}^{*}$ & SE & p & $\mathrm{R}^{2}$ & $\mathrm{~b}$ & SE & p & $\mathrm{R}^{2}$ & $\mathrm{~b}$ & SE & p & $\mathrm{R}^{2}$ \\
\hline $\mathrm{CA}$ & 0.44 & - & .08 & $<0.001 *$ & 03 & - & - & - & - & - & - & - & - \\
\hline Frn & -0.35 & -0.33 & .05 & $<0.001 *$ & 05 & -0.36 & .06 & $<0.001 *$ & 05 & -0.29 & .07 & $<0.001 *$ & 03 \\
\hline Fms & -0.31 & -0.28 & .04 & $<0.001 *$ & 04 & -0.28 & .06 & $<0.001 *$ & 04 & -0.30 & .06 & $<0.001 *$ & 04 \\
\hline Fmc & -0.39 & -0.35 & .04 & $<0.001 *$ & 07 & -0.38 & .06 & $<0.001 *$ & 06 & -0.31 & .07 & $<0.001 *$ & 04 \\
\hline Pst & -0.47 & -0.44 & .04 & $<0.001 *$ & 11 & -0.43 & .05 & $<0.001 *$ & 09 & -0.47 & .06 & $<0.001 *$ & 11 \\
\hline $\mathrm{Ngt}$ & -0.60 & -0.57 & .05 & $<0.001 *$ & 14 & -0.50 & .06 & $<0.001 *$ & 10 & -0.69 & .07 & $<0.001 *$ & 18 \\
\hline Brd & -0.50 & -0.48 & .04 & $<0.001 *$ & 11 & -0.45 & .06 & $<0.001 *$ & 09 & -0.52 & .06 & $<0.001 *$ & 12 \\
\hline Rfl & -0.32 & -0.31 & .05 & $<0.001 *$ & 04 & -0.27 & .06 & $<0.001 *$ & 03 & -0.36 & .07 & $<0.001 *$ & 06 \\
\hline Dst & -0.26 & -0.23 & .04 & $<0.001 *$ & 03 & -0.30 & .06 & $<0.001 *$ & 04 & -0.14 & .06 & $<0.05 *$ & 01 \\
\hline Agg & -0.25 & -0.23 & .04 & $<0.001 *$ & 04 & -0.22 & .05 & $<0.001 *$ & 03 & -0.25 & .06 & $<0.001 *$ & 03 \\
\hline Exp & 0.01 & 0.03 & .04 & 0.72 & 00 & 0.05 & .05 & 0.36 & 00 & 0.01 & .06 & 0.80 & 00 \\
\hline D14 & 0.65 & 0.63 & .04 & $<0.001 *$ & 22 & 0.61 & .05 & $<0.001 *$ & 19 & 0.67 & .05 & $<0.001 *$ & 24 \\
\hline
\end{tabular}

Note. $\mathrm{b}=$ unstandardized regression (slope) coefficient, $\mathrm{b}^{*}=$ unstandardized regression (slope) coefficient corrected for CA; $\mathrm{SE}=$ standard error, $\mathrm{p}=\mathrm{p}$-value, $\mathrm{R}^{2}=$ variance explained, represented here as percentage. Of note, $\mathrm{SE}, \mathrm{p}$ and $\mathrm{R}^{2}$ belong to the effects that are not corrected for $\mathrm{CA} . \mathrm{CA}=$ childhood adversity, $\mathrm{CA}+=$ adolescents with $\mathrm{CA}, \mathrm{CA}-=$ adolescents without $\mathrm{CA}$; Frn = friendship support Fms = family support Fmc = family cohesion; $\mathrm{Pst}=$ positive self-esteem; $\mathrm{Ngt}=$ negative self-esteem; $\mathrm{Brd}=$ brooding; Rfl = reflection; Dst = distress tolerance; Agg = aggression; Exp = expressive suppression; D14 = age-14 distress.

Table 3

Single moderation and mediation effects of the RFs without correction for gender; corrected for multiple testing

\begin{tabular}{|c|c|c|c|c|c|c|c|c|}
\hline & \multicolumn{4}{|c|}{ Moderation: interaction effect } & \multirow[b]{2}{*}{$\mathrm{b}$} & \multicolumn{3}{|c|}{ Mediation: indirect effect } \\
\hline & $\mathrm{b}$ & $\mathrm{SE}$ & $\mathrm{p}$ & $\% R^{2}$ & & SE & $\mathrm{p}$ & $\mathrm{MC} \mathrm{CI}$ \\
\hline Frn & -0.06 & 0.10 & 0.79 & 07 & 0.04 & 0.02 & $<0.01 *$ & $.01-.08$ \\
\hline Fms & 0.02 & 0.09 & 0.83 & 07 & 0.05 & 0.02 & $<0.01 *$ & $.02-.08$ \\
\hline Fmc & -0.07 & 0.09 & 0.78 & 08 & 0.13 & 0.02 & $<0.001 *$ & $.08-.18$ \\
\hline Pst & 0.05 & 0.08 & 0.79 & 13 & 0.13 & 0.03 & $<0.001 *$ & $.08-.18$ \\
\hline Ngt & 0.20 & 0.09 & 0.30 & 15 & 0.11 & 0.03 & $<0.001 *$ & $.06-.17$ \\
\hline Brd & 0.07 & 0.09 & 0.78 & 13 & 0.07 & 0.03 & $<0.01 *$ & $.03-.12$ \\
\hline Rfl & 0.09 & 0.09 & 0.78 & 07 & 0.02 & 0.02 & 0.23 & $-.01-.05$ \\
\hline Dst & -0.17 & 0.09 & 0.30 & 06 & 0.06 & 0.02 & $<0.001 *$ & $.03-.09$ \\
\hline Agg & 0.03 & 0.08 & 0.82 & 06 & 0.05 & 0.02 & $<0.01 *$ & $.02-.09$ \\
\hline Exp & 0.03 & 0.08 & 0.82 & 03 & -0.01 & 0.01 & 0.41 & $-.02-.01$ \\
\hline D14 & -0.06 & 0.07 & 0.78 & 23 & 0.17 & 0.04 & $<0.001 *$ & $.10-.25$ \\
\hline
\end{tabular}

Note. $\mathrm{b}=$ unstandardized regression (slope) coefficient, $\mathrm{SE}=$ standard error, $\mathrm{p}=\mathrm{p}$-value, $\% \mathrm{R}^{2}=$ percentage variance explained. MC CI = Monetcarlo confidence interval. Frn = friendship support; Fms = family support; Fmc = family cohesion; Pst = 
positive self-esteem; $\mathrm{Ngt}=$ negative self-esteem; $\mathrm{Brd}=$ brooding; $\mathrm{Rfl}=$ reflection; $\mathrm{Dst}=$ distress tolerance; $\mathrm{Agg}=$ aggression; Exp $=$ expressive suppression; D14 = age-14 distress .

Table 4

Multiple main effects of the RFs without correction for gender, for the whole sample, $\mathrm{CA}+$ and the CA-group

\begin{tabular}{|c|c|c|c|c|c|c|c|c|c|c|}
\hline & \multicolumn{4}{|c|}{ Whole sample } & \multicolumn{3}{|c|}{$\mathrm{CA}+$} & \multicolumn{3}{|c|}{$C A-$} \\
\hline & $\mathrm{b}$ & $b^{*}$ & $\mathrm{SE}$ & $\mathrm{p}$ & $\mathrm{b}$ & $\mathrm{SE}$ & $\mathrm{p}$ & $\mathrm{b}$ & $\mathrm{SE}$ & $\mathrm{p}$ \\
\hline $\mathrm{CA}$ & 0.25 & - & .07 & $<0.001 *$ & - & - & - & - & - & - \\
\hline Frn & -0.07 & -0.07 & .05 & 0.15 & -0.15 & .07 & $<0.05^{*}$ & 0.04 & .08 & 0.56 \\
\hline Fms & -0.03 & -0.05 & .06 & 0.55 & 0.02 & .08 & 0.85 & -0.14 & .08 & 0.08 \\
\hline Fmc & -0.12 & -0.09 & .06 & $<0.05^{*}$ & -0.17 & .08 & $<0.05^{*}$ & 0.02 & .08 & 0.78 \\
\hline Pst & -0.18 & -0.17 & .05 & $<0.001 *$ & -0.19 & .07 & $<0.01 *$ & -0.15 & .07 & $<0.05 *$ \\
\hline $\mathrm{Ngt}$ & -0.20 & -0.21 & .07 & $<0.01 *$ & -0.06 & .09 & 0.50 & -0.41 & .10 & $<0.001 *$ \\
\hline Brd & -0.18 & -0.18 & .06 & $<0.01 *$ & -0.17 & .09 & $<0.05 *$ & -0.18 & .09 & $<0.05 *$ \\
\hline Rfl & -0.01 & -0.02 & .06 & 0.81 & 0.01 & .08 & 0.91 & -0.09 & .08 & 0.26 \\
\hline Dst & -0.08 & -0.06 & .04 & 0.07 & -0.13 & .06 & $<0.05^{*}$ & 0.01 & .06 & 0.82 \\
\hline Agg & -0.11 & -0.10 & .04 & $<0.01 *$ & -0.13 & .05 & $<0.01 *$ & -0.06 & .06 & 0.35 \\
\hline Exp & 0.05 & 0.06 & .04 & 0.14 & 0.06 & .05 & 0.26 & 0.05 & .05 & 0.31 \\
\hline$\% \mathrm{R}^{2}$ & & & & $19 \%$ & & & $17 \%$ & & & $21 \%$ \\
\hline
\end{tabular}

Note. $\mathrm{b}=$ unstandardized regression (slope) coefficient, $\mathrm{b}^{*}=$ unstandardized regression (slope) coefficient corrected for $\mathrm{CA}$; $\mathrm{SE}=$ standard error, $\mathrm{p}=\mathrm{p}$-value, $\% \mathrm{R}^{2}=$ percentage variance explained. Of note, $\mathrm{SE}, \mathrm{p}$ and $\% \mathrm{R}^{2}$ belong to the effects that are not corrected for $\mathrm{CA}$. $\mathrm{CA}=$ childhood adversity, $\mathrm{CA}+=$ adolescents with $\mathrm{CA}, \mathrm{CA}-=$ adolescents without $\mathrm{CA}$; Frn $=$ friendship support; Fms = family support; Fmc $=$ family cohesion; Pst $=$ positive self-esteem; Ngt $=$ negative self-esteem; Brd = brooding; $\mathrm{Rfl}=$ reflection $;$ Dst $=$ distress tolerance $;$ Agg $=$ aggression $;$ Exp $=$ expressive suppression . 


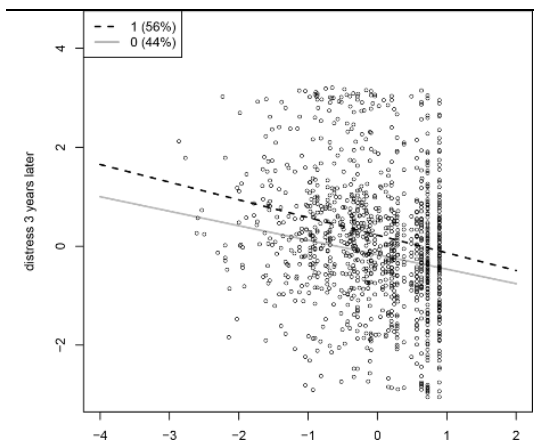

Friendship support

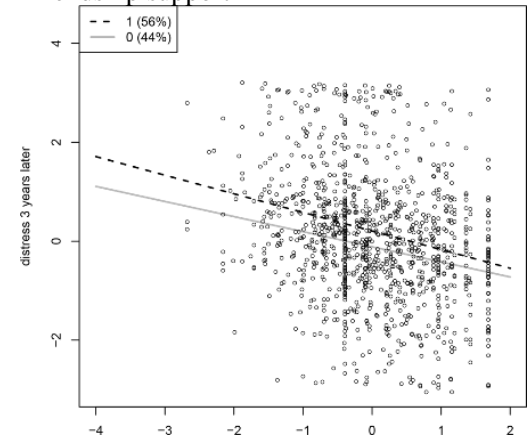

Family cohesion

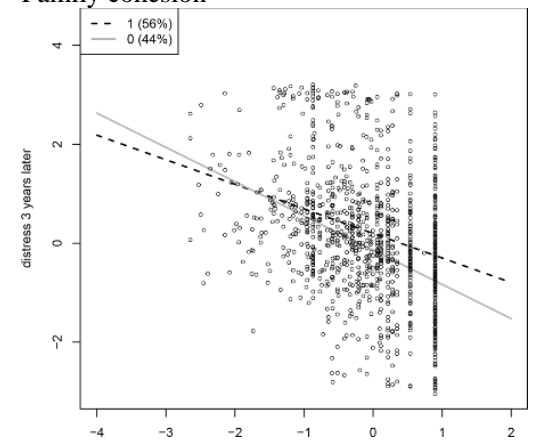

Negative self-esteem

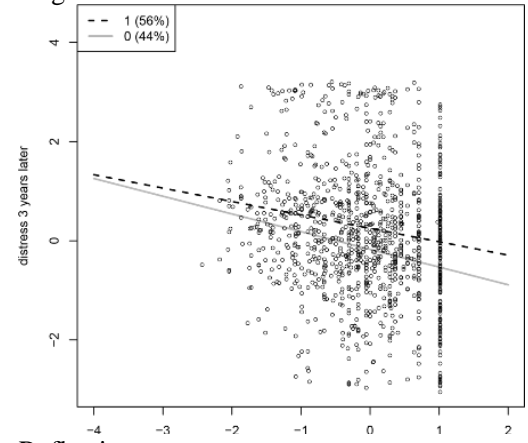

Reflection

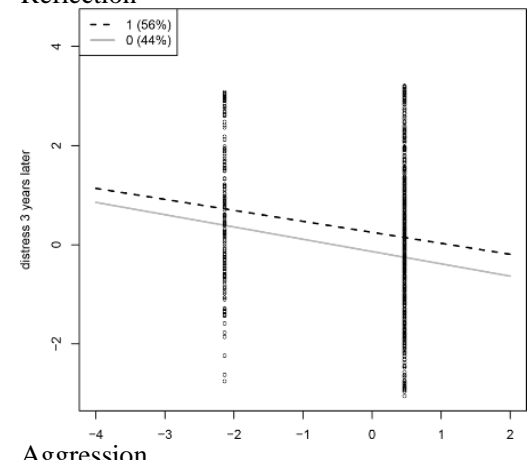

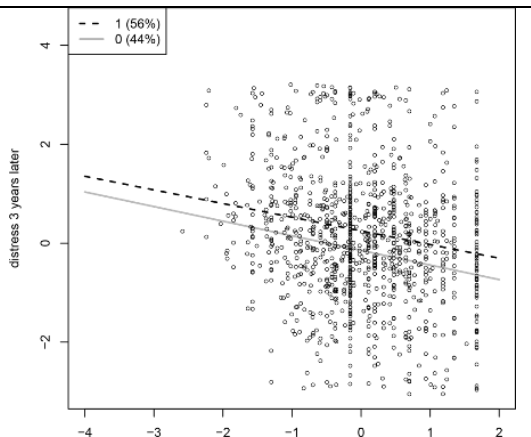

Family support

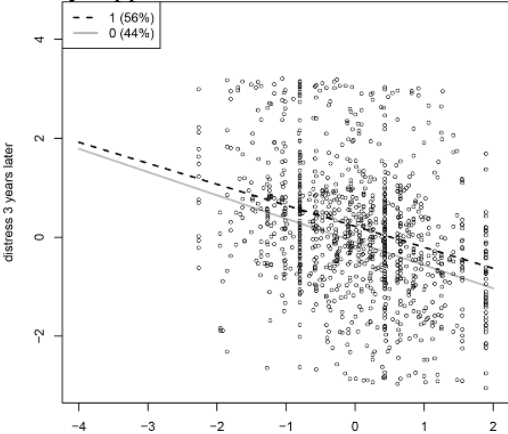

Positive self-esteem

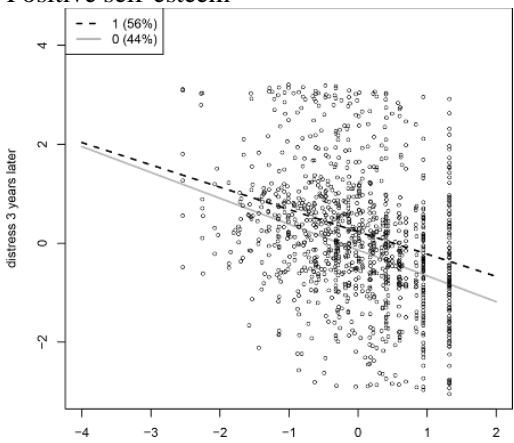

Brooding

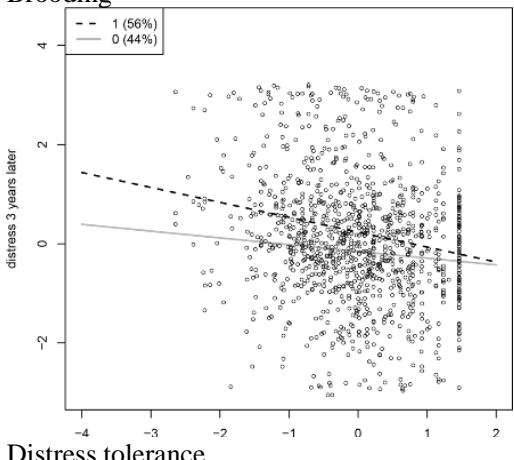

Distress tolerance

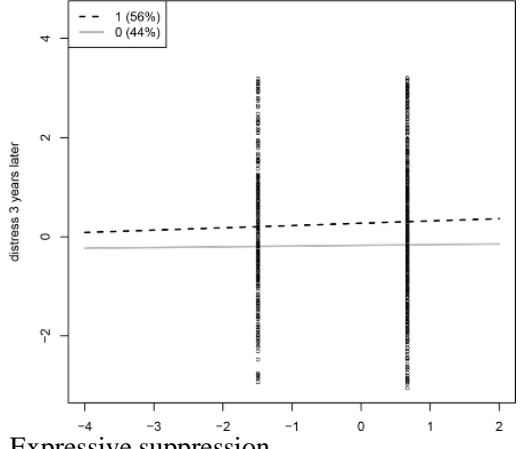

Figure 1. The moderation effects of the RFs for the relationship between CA and age-17-distress. Dashed line = $\mathrm{CA}+$, solid line $=\mathrm{CA}-$. 


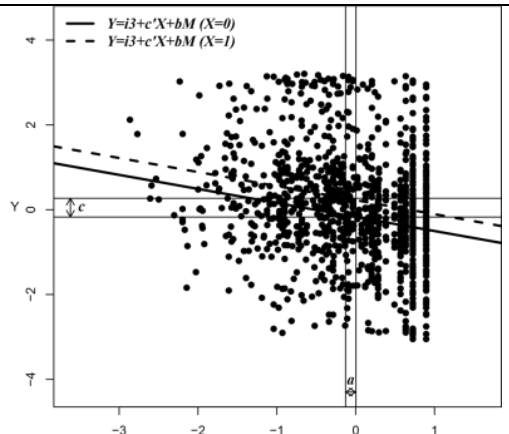

${ }^{-3}$ Friendship $^{-1}$ support

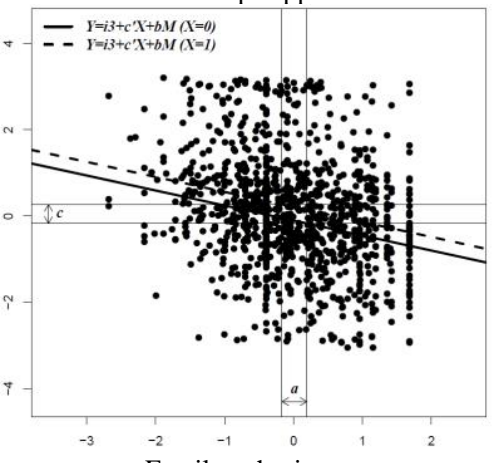

Family cohesion
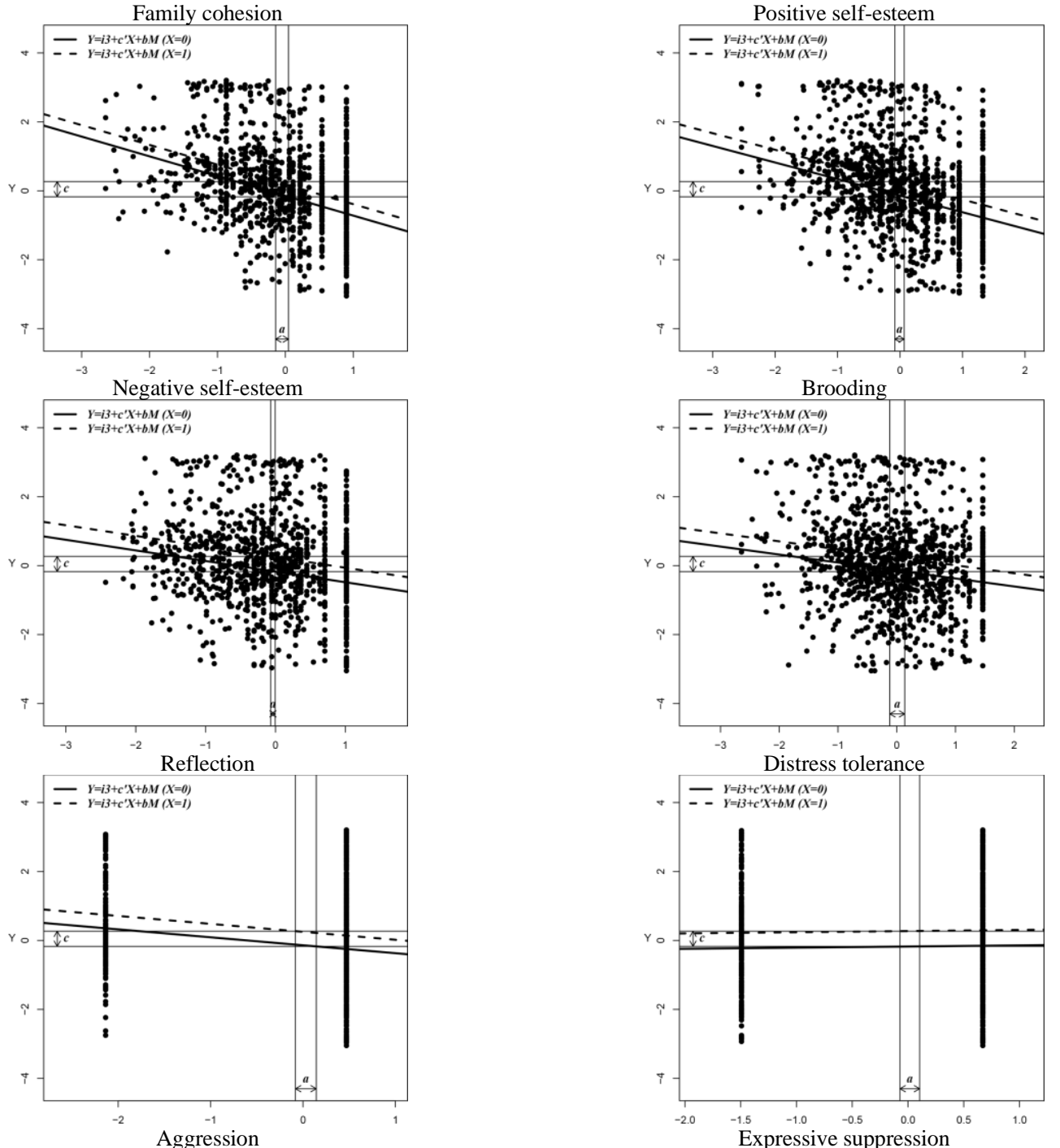

Figure 2. The mediation effects of the RFs for the relationship between CA and age-17-distress. Dashed line $=$ $\mathrm{CA}+$, solid line $=\mathrm{CA}-$. 


\section{Supplement III}

\begin{tabular}{|c|c|}
\hline Package (version number) & Reference \\
\hline \multirow[t]{4}{*}{$\operatorname{car}(3.0-2)$} & John Fox and Sanford Weisberg (2011). An $\{\mathrm{R}\}$ \\
\hline & Companion to Applied Regression, Second Edition. \\
\hline & Thousand Oaks CA: Sage. ${ }^{64}$ \\
\hline & http://socserv.socsci.mcmaster.ca/jfox/Books/Companion \\
\hline \multirow[t]{3}{*}{ dplyr (0.7.7) } & Hadley Wickham, Romain François, Lionel Henry and \\
\hline & Kirill Müller (2018). dplyr: A Grammar of Data \\
\hline & Manipulation. https://CRAN.R- \\
\hline \multirow{2}{*}{ foreign $(0.8-70)$} & 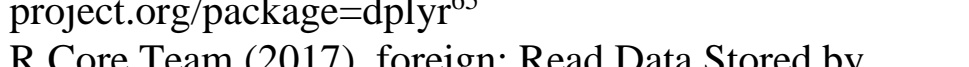 \\
\hline & $\begin{array}{l}\text { 'Minitab', 'S', 'SAS', 'SPSS', 'Stata', 'Systat', 'Weka', } \\
\text { 'dBase', .... R package version 0.8-70. https://CRAN.R- } \\
\text { project.org/package=foreign }{ }^{66}\end{array}$ \\
\hline \multirow[t]{4}{*}{ lavaan $(0.6-4)$} & Yves Rosseel (2012). lavaan: An R Package for \\
\hline & Structural Equation Modeling. Journal of Statistical \\
\hline & Software, 48(2), 1-36. \\
\hline & http://www.jstatsoft.org/v48/i02/. ${ }^{33}$ \\
\hline mice $(3.5 .0)$ & $\begin{array}{l}\text { Stef van Buuren, Karin Groothuis-Oudshoorn (2011). } \\
\text { mice: Multivariate Imputation by Chained Equations in } \\
\text { R. Journal of Statistical Software, } 45(3), 1-67 . \\
\text { https://www.jstatsoft.org/v45/i03/. }\end{array}$ \\
\hline \multirow[t]{2}{*}{ pastecs $(1.3 .21)$} & Philippe Grosjean and Frederic Ibanez (2018). pastecs: \\
\hline & $\begin{array}{l}\text { Package for Analysis of Space-Time Ecological Series. } \\
\text { https://CRAN.R-project.org/package=pastecs }\end{array}$ \\
\hline \multirow[t]{2}{*}{ rockchalk (1.8.129) } & Paul E. Johnson (2018), rockchalk: Regression \\
\hline & $\begin{array}{l}\text { Estimation and Presentation. R package version 1.8.129. } \\
\text { https://CRAN.R-project.org/package=rockchalk }\end{array}$ \\
\hline \multirow[t]{2}{*}{ semTools $(0.5-1.933)$} & Jorgensen, T. D., Pornprasertmanit, S., Schoemann, A. \\
\hline & $\begin{array}{l}\text { M., \& Rosseel, Y. (2019). semTools: Useful tools for } \\
\text { structural equation modeling. R package version 0.5- } \\
\text { 1.933. Retrieved from https://CRAN.R- } \\
\text { project.org/package=semTools }{ }^{69}\end{array}$ \\
\hline \multirow[t]{3}{*}{ sjPlot (2.6.2) } & Lüdecke D (2018). sjPlot: Data Visualization for \\
\hline & Statistics in Social Science. R package version 2.6.2. doi: \\
\hline & $\begin{array}{l}\text { 10.5281/zenodo.1308157. https://CRAN.R- } \\
\text { project.org/package }=\text { sjPlot }^{70}\end{array}$ \\
\hline
\end{tabular}




\section{Supplement IV}

\section{Expected and achieved power}

In line with our preregistered review, we would have expected a low interaction effect size for the moderation effect of the RF and CA (i.e. $\mathrm{f}^{2}=0.01$ ). Given the total sample size of 1130 , and $\alpha / \beta$ ratio of 1 , three predictors in total ( 2 main effects, 1 interaction effect) and 1 predictor being tested (i.e. the interaction effect) our analyses would have had a high amount of power $(=.94)$. For the indirect effect of the mediation models, we would have assumed a moderate, positive correlation between CA and distress at age $17(\sim+0.30)$, a small, negative correlation between $\mathrm{CA}$ and the RF ( -0.10), and a moderate, negative correlation between the RF and distress at age $17(\sim-0.30)$. We specified a standard deviation of 1 for the RF and age-17 distress, as most of our RFs and the distress scores were derived form a standardized distribution, and a standard deviation of 0.5 for the $\mathrm{CA}$ variable as this one is binary (alongside, 1000 replications with 100000 Monte Carlo draws, a seed of 4444 and 95\% confidence interval). Accordingly, the indirect mediation effects would also have had a high amount of power $(=.91)$. We additionally computed the post-hoc power that actually was achieved, which can be found in Table 2 and clearly reflects our results pattern.

Table 2

\begin{tabular}{lll}
\hline & Moderation & Mediation \\
\hline Friendship support & $\triangle \mathrm{R}^{2}=0.0004 ; 0.10$ & $0.80^{* * *}$ \\
Family support & $\triangle \mathrm{R}^{2}=0.0000 ; 0.06$ & $0.88^{* * *}$ \\
Family cohesion & $\triangle \mathrm{R}^{2}=0.0005 ; 0.12$ & $1.00^{* * *}$ \\
Positive self-esteem & $\triangle \mathrm{R}^{2}=0.0003 ; 0.09$ & $1.00^{* * *}$ \\
Negative self-esteem & $\triangle \mathrm{R}^{2}=0.0034 ; 0.50^{*}$ & $0.98^{* * *}$ \\
Brooding & $\triangle \mathrm{R}^{2}=0.0005 ; 0.12$ & $0.84^{* * *}$ \\
Reflection & $\triangle \mathrm{R}^{2}=0.0007 ; 0.15$ & 0.26 \\
Distress tolerance & $\triangle \mathrm{R}^{2}=0.0031 ; 0.46$ & $1.00^{* * *}$ \\
Aggression & $\triangle \mathrm{R}^{2}=0.0001 ; 0.06$ & $0.97^{* * *}$ \\
Expressive suppression & $\triangle \mathrm{R}^{2}=0.0002 ; 0.07$ & 0.12 \\
Age-14 distress & $\triangle \mathrm{R}^{2}=0.0005 ; 0.12$ & $1.00^{* * *}$ \\
\hline
\end{tabular}

Note. The post-hoc power analyses for the interaction effects of the single moderation models were computed with GPower ${ }^{71}$, with the following specifications: model $=$ linear multiple regression model $\mathrm{R}^{2}$ increase, analysis = post-hoc: compute achieved power, $\mathrm{N}=1130, \alpha=0.05, \mathrm{~N}$ of predictors $=3$ ( 2 main effects, 1 interaction effect), tested predictors $=1$, we then used the increase in $\mathrm{R}^{2}$ after taking the interaction effect into account to calculate the $\mathrm{f}^{2}$ effect size for the interaction effect. The post-hoc power analyses for the indirect effect of the single mediation models were conducted with the power analysis app written by Schoemann, Boulton, \& Short ${ }^{72}$, with the following specifications: $\mathrm{N}=1130,1000$ replications, 100000 Monte Carlo Draws per replication, seed $=$ 4444, 95\% CI, and specified the CA-RF, CA-distress, and RF-distress correlations (alongside the standard deviations of the three variables). ${ }^{* * *}$ power $\geq 0.80,{ }^{* *}$ power $\geq 0.65, *$ power $\geq 0.50$ 


\section{Supplement V}

For the models without gender and age-14 distress, none of the RFs had a variance inflation factor (VIF) above 3, regardless of investigating the $\mathrm{CA}+$, the $\mathrm{CA}$ - or the whole sample (see Table 3). For the models with gender, but without age-14 distress, none of the RFs had a VIF above 3, regardless of investigating the $\mathrm{CA}+$, the $\mathrm{CA}$ - or the whole sample. For the models with gender and age-14 distress, negative self-esteem had a VIF above 3 in the whole sample as well as in the CA+ group. Yet, negative self-esteem had a VIF lower than 3 in the CAgroup. Moreover, for the models with gender and age-14 distress, age-14 distress had a VIF above 3 in all three samples (i.e. the whole sample, the CA+ and the CA-group). Importantly, when taking the square root of the VIFs, none was bigger than 2, which indicates that even those variables that have a VIF above 3, are only borderline cases for multicollinearity.

Table 3

Variance inflation factors

\begin{tabular}{|c|c|c|c|c|c|c|c|c|c|c|c|c|c|}
\hline & $\mathrm{CA}$ & gender & Frn & Fms & Fmc & Pst & $\mathrm{Ngt}$ & Brd & Rfl & Dst & Agg & Exp & D14 \\
\hline WS & 1.08 & - & 1.22 & 1.90 & 2.11 & 1.82 & 2.40 & 2.27 & 1.66 & 1.15 & 1.13 & 1.06 & - \\
\hline $\mathrm{CA}+$ & - & - & 1.21 & 1.95 & 2.13 & 1.83 & 2.54 & 2.43 & 1.80 & 1.16 & 1.11 & 1.07 & - \\
\hline CA- & - & - & 1.29 & 1.82 & 1.90 & 1.72 & 2.20 & 2.09 & 1.54 & 1.10 & 1.15 & 1.06 & - \\
\hline WS & 1.08 & 1.23 & 1.23 & 1.91 & 2.11 & 1.85 & 2.41 & 2.27 & 1.75 & 1.17 & 1.17 & 1.06 & - \\
\hline $\mathrm{CA}+$ & - & 1.18 & 1.22 & 1.95 & 2.14 & 1.85 & 2.56 & 2.43 & 1.84 & 1.17 & 1.16 & 1.08 & - \\
\hline CA- & - & 1.33 & 1.30 & 1.83 & 1.90 & 1.76 & 2.20 & 2.09 & 1.71 & 1.13 & 1.19 & 1.07 & - \\
\hline WS & 1.08 & 1.24 & 1.27 & 1.91 & 2.13 & 1.87 & 3.03 & 2.49 & 1.76 & 1.17 & 1.21 & 1.06 & 3.52 \\
\hline $\mathrm{CA}+$ & - & 1.20 & 1.29 & 1.95 & 2.17 & 1.88 & 3.23 & 2.62 & 1.85 & 1.18 & 1.21 & 1.08 & 3.79 \\
\hline CA- & - & 1.33 & 1.32 & 1.83 & 1.92 & 1.78 & 2.74 & 2.34 & 1.73 & 1.13 & 1.21 & 1.07 & 3.13 \\
\hline
\end{tabular}

Note. $\mathrm{WS}=$ whole sample, $\mathrm{CA}+=$ with childhood adversity, $\mathrm{CA}-=$ without childhood adversity; Frn $=$ friendship support; Fms = family support; Fmc = family cohesion; Ngt = negative self-esteem; Pst = positive self-esteem; Brd = brooding; Rfl = reflection; Dst = distress tolerance; Agg = aggression; Exp = expressive suppression; D14 = age-14 distress. When taking the square root of the variance inflation factors, none was bigger than 2, which additionally underpins the absence of multicollinearity. 


\section{Supplement VI}

This Supplement contains the multiple moderation and mediation results, with and without correction for gender. The right panel of Table 7 corresponds to Figure 3 in the main manuscript. The right panel of Table 8 corresponds to Figure 3 in the main manuscript.

Table 7

Multiple moderation effects of the RFs without and with correction for gender

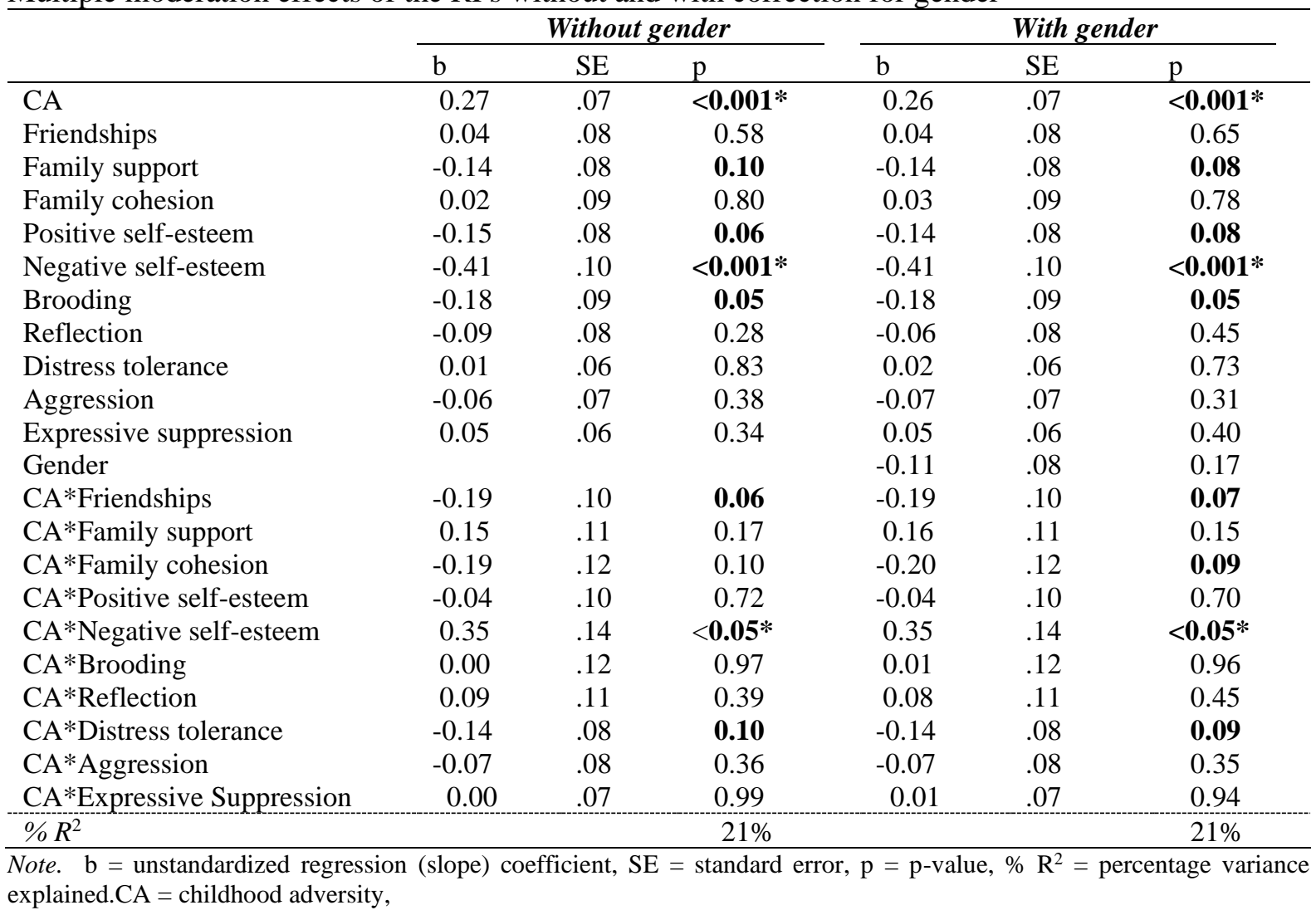

Table 8

Multiple mediation effects of the RFs with and without correction for gender

\begin{tabular}{|c|c|c|c|c|c|c|c|}
\hline \multirow[b]{2}{*}{ Path } & & \multicolumn{3}{|c|}{ Without gender } & \multicolumn{3}{|c|}{ With gender } \\
\hline & & EST & $\mathrm{p}$ & $\% R^{2}$ & EST & $\mathrm{P}$ & $\% R^{2}$ \\
\hline $\mathrm{CA}^{1} \rightarrow$ age 17 -distress & $\mathrm{c}$ & 0.44 & $<0.001 *$ & & 0.43 & $<0.001 *$ & \\
\hline $\mathrm{CA} \rightarrow$ age17-distress & $c^{\prime}$ & 0.25 & $<0.01 *$ & & 0.25 & $<0.01 *$ & \\
\hline Friendships $\rightarrow$ age17-distress & b1 & -0.07 & 0.17 & & -0.07 & 0.14 & \\
\hline Family sup. $\rightarrow$ age17-distress & $\mathrm{b} 2$ ' & -0.05 & 0.41 & & -0.05 & 0.37 & \\
\hline Family coh. $\rightarrow$ age17-distress & b3' & -0.09 & 0.10 & & -0.09 & 0.09 & \\
\hline Pos. SE $\rightarrow$ age 17 -distress & b4' & -0.17 & $<0.01 *$ & & -0.16 & $<0.01 *$ & \\
\hline Neg. SE $\rightarrow$ age17-distress & b5 & -0.21 & $<0.01 *$ & & -0.20 & $<0.01 *$ & \\
\hline Brooding $\rightarrow$ age 17-distress & b6' & -0.18 & $<0.01 *$ & & -0.18 & $<0.01 *$ & \\
\hline Reflection $\rightarrow$ age17-distress & b7 & -0.02 & 0.76 & & 0.00 & 0.98 & \\
\hline Distress tol. $\rightarrow$ age17-distress & $\mathrm{b} 8{ }^{\prime}$ & -0.06 & 0.14 & & -0.05 & 0.20 & \\
\hline Aggression $\rightarrow$ age 17-distress & b9' & -0.10 & $<0.05 *$ & & -0.11 & $<0.01 *$ & \\
\hline Exp. sup. $\rightarrow$ age 17 -distress & b10' & 0.06 & 0.08 & & 0.06 & 0.09 & \\
\hline Gender $\rightarrow$ age 17 -distress & $\operatorname{cov}$ & - & - & & -0.11 & 0.18 & \\
\hline $\mathrm{CA}^{1} \rightarrow$ Friendships & a1 & -0.13 & $<0.01 *$ & 0.7 & -0.13 & $<0.01 *$ & 0.7 \\
\hline $\mathrm{CA}^{1} \rightarrow$ Family sup. & $\mathrm{a} 2$ & -0.16 & $<0.01 *$ & 0.9 & -0.17 & $<0.01 *$ & 0.9 \\
\hline $\mathrm{CA}^{1} \rightarrow$ Family coh. & a3 & -0.36 & $<0.001 *$ & 4.3 & -0.36 & $<0.001 *$ & 4.4 \\
\hline $\mathrm{CA}^{1} \rightarrow$ Pos. SE & a4 & -0.28 & $<0.001 *$ & 2.3 & -0.27 & $<0.001 *$ & 6.3 \\
\hline
\end{tabular}




\begin{tabular}{|c|c|c|c|c|c|c|c|}
\hline $\mathrm{CA}^{1} \rightarrow$ Neg. SE & a5 & -0.19 & $<0.001 *$ & 1.4 & -0.18 & $<0.001 *$ & 5.5 \\
\hline $\mathrm{CA}^{1} \rightarrow$ Brooding & $\mathrm{a} 6$ & -0.15 & $<0.01 *$ & 0.8 & -0.14 & $<0.01 *$ & 5.8 \\
\hline $\mathrm{CA}^{1} \rightarrow$ Reflection & a7 & -0.07 & 0.19 & 0.2 & -0.04 & 0.36 & 10.0 \\
\hline $\mathrm{CA}^{1} \rightarrow$ Distress tol. & $\mathrm{a} 8$ & -0.26 & $<0.001 *$ & 2.0 & -0.24 & $<0.001 *$ & 6.0 \\
\hline $\mathrm{CA}^{1} \rightarrow$ Aggression & a9 & -0.23 & $<0.001 *$ & 1.3 & -0.24 & $<0.001 *$ & 3.2 \\
\hline $\mathrm{CA}^{1} \rightarrow$ Exp. sup. & a10 & -0.18 & $<0.01 *$ & 0.8 & -0.18 & $<0.01 *$ & 1.5 \\
\hline indirect effect friendships & $\mathrm{a} 1 * \mathrm{~b} 1{ }^{\prime}$ & 0.009 & 0.22 & & 0.010 & 0.20 & \\
\hline indirect effect family sup. & $\mathrm{a} 2 * \mathrm{~b} 2{ }^{\prime}$ & 0.008 & 0.44 & & 0.008 & 0.40 & \\
\hline indirect effect family coh. & a3*b3' & 0.034 & 0.11 & & 0.034 & 0.10 & \\
\hline indirect effect pos. SE & $\mathrm{a} 4 * \mathrm{~b} 4{ }^{\prime}$ & 0.048 & $<0.01 *$ & & 0.043 & $<0.01 *$ & \\
\hline indirect effect neg. SE & $\mathrm{a} 5 * \mathrm{~b} 5$ & 0.039 & $<0.05 *$ & & 0.035 & $<0.05 *$ & \\
\hline indirect effect brooding & $\mathrm{a} 6 * \mathrm{~b} 6$ & 0.027 & $<0.05 *$ & & 0.024 & 0.06 & \\
\hline indirect effect reflection & $\mathrm{a} 7 * \mathrm{~b} 7$ & 0.001 & 0.81 & & -0.000 & 0.98 & \\
\hline indirect effect distress tol. & $\mathrm{a} 8 * \mathrm{~b} 8$ & 0.015 & 0.15 & & 0.013 & 0.21 & \\
\hline indirect effect aggression & a9*b9' & 0.023 & $<0.05 *$ & & 0.026 & $<0.05 *$ & \\
\hline indirect effect exp. sup. & $\mathrm{a} 10 * \mathrm{~b} 10$ & -0.011 & 0.15 & & -0.011 & 0.15 & \\
\hline Total indirect effect & ind & 0.193 & $<0.001 *$ & & 0.183 & $<0.001 *$ & \\
\hline Total indirect RF effect & ind RF & 0.204 & $<0.001 *$ & & 0.194 & $<0.001 *$ & \\
\hline Total effect & c'+ind & 0.441 & $<0.001 *$ & 20.0 & 0.429 & $<0.001 *$ & 20.0 \\
\hline \multicolumn{8}{|c|}{$\begin{array}{l}\text { Note. } \mathrm{c}=\text { direct effect (that is the effect of CA alone, and if included gender). } \mathrm{c}^{\prime}=\text { corrected direct effect (that is the effect } \\
\text { of CA, after taking into account all RFs, and if included gender). } \% R^{2}=\text { percentage variance explained. }{ }^{1}=\text { This path is in } \\
\text { the second model also controlled for gender. Sup. = support, coh. = cohesion, SE = self-esteem, pos. = positive, neg. }= \\
\text { negative, tol. = tolerance, Exp. sup. = Expressive suppression, cov }=\text { covariate. Total indirect effect = includes all } 10 \mathrm{RFs} \text {. } \\
\text { Total indirect RF effect = includes only those RFs that qualified as RF mediators (i.e. those with a negative a- and b-path). } \\
\text { Total effect = that is the corrected direct effect of CA, plus the total indirect effect that goes via the RFs, and if included } \\
\text { gender. Please note, the reported estimates and p-values are derived from the maximum likelihood models with bootstrapped } \\
\text { standard errors. }\end{array}$} \\
\hline
\end{tabular}

